\title{
MEAN CURVATURE FLOW OF KILLING GRAPHS
}

\author{
J. H. LIRA AND G. A. WANDERLEY
}

Abstract. We study a Neumann problem related to the evolution of graphs under mean curvature flow in Riemannian manifolds endowed with a Killing vector field. We prove that in a particular case these graphs converge to a trivial minimal graph which contacts the cylinder over the domain orthogonally along its boundary.

\section{INTRODUCTION}

Let $M$ be an $(n+1)$-dimensional Riemannian manifold endowed with a Killing vector field $Y$. Suppose that the distribution orthogonal to $Y$ is of constant rank and integrable. Given an integral leaf $P$ of that distribution, let $\Omega \subset P$ be a bounded domain with regular boundary $\Gamma=\partial \Omega$. Let $\vartheta: \mathbb{I} \times \bar{\Omega} \rightarrow M$ be the flow generated by $Y$ with initial values in $M$, where $\mathbb{I}$ is a maximal interval of definition. In geometric terms, the ambient manifold is a warped product $M=P \times_{1 / \sqrt{\gamma}} \mathbb{I}$ where $\gamma=1 /|Y|^{2}$.

Given $T \in[0,+\infty)$, let $u: \bar{\Omega} \times[0, T) \rightarrow \mathbb{I}$ be a smooth function. Fixing this notation, the Killing graph of $u(\cdot, t), t \in[0, T)$, is the hypersurface $\Sigma_{t} \subset M$ parametrized by the map

$$
X(t, x)=\vartheta(u(x, t), x), \quad x \in \bar{\Omega} .
$$

Notice that this definition could be slightly more general if we suppose that the coordinates of $x \in \bar{\Omega}$ change with the parameter $t \in[0, T)$. To abolish this possibility is equivalent to ruling out tangential diffeomorphisms of $\Omega$.

The Killing cylinder $K$ over $\Gamma$ is in turn defined by

$$
K=\{\vartheta(s, x): s \in \mathbb{I}, x \in \Gamma\} .
$$

Let $N$ be a unit normal vector field along $\Sigma_{t}$. In what follows, we denote by $H$ the mean curvature of $\Sigma_{t}$ with respect to the orientation given by $N$. We are then concerned with establishing conditions for longtime existence of a prescribed mean curvature flow of the form

$$
\begin{gathered}
\frac{\partial X}{\partial t}=(n H-\mathcal{H}) N, \\
X(0, \cdot)=\vartheta\left(u_{0}(\cdot), \cdot\right),
\end{gathered}
$$

for given functions $u_{0}: \bar{\Omega} \rightarrow \mathbb{R}$ and $\mathcal{H}: \bar{\Omega} \rightarrow \mathbb{R}$. In order to define boundary conditions for the evolution problem (1.2) we consider a function $\phi \in C^{\infty}(\Gamma)$ such that $|\phi| \leq \phi_{0}<1$ for some positive constant $\phi_{0}$. Let $\nu$ be the inward unit normal

Received by the editors October 1, 2012 and, in revised form, March 9, 2013.

2010 Mathematics Subject Classification. Primary 53C42, 53C44.

The first author was partially supported by CNPq and PRONEX/FUNCAP.

The second author was partially supported by CAPES. 
vector field along $K$. We impose the following Neumann condition associated to (1.2):

$$
\left.\langle N, \nu\rangle\right|_{\partial \Sigma_{t}}=\phi
$$

where $\langle\cdot, \cdot\rangle$ denotes the Riemannian metric in $M$.

The main result in this paper may be stated as follows.

Theorem 1. There exists a unique solution $u: \bar{\Omega} \times[0, \infty) \rightarrow \mathbb{I}$ to the problem (1.2)(1.4). Moreover, if $\phi=0$ and $\mathcal{H}=0$, the functions $u(\cdot, t)$ converge to a constant function as $t \rightarrow \infty$.

Theorem 1]extends Theorem 1.1 in [3] as well as Theorem 2.4 in [2] and Theorem 2.4 in [1] in a twofold way. The corresponding theorems in [3] and 2] concern evolution of graphs in Euclidean space whereas [1] deals with the case of graphs in Riemannian product spaces of the form $P \times \mathbb{R}$. Moreover those earlier results hold only for the case when the prescribed mean curvature is $\mathcal{H}=0$. An existence result for evolution of graphs in Euclidean space by the Gauss-Kronecker curvature under Neumann boundary conditions is proved in [8]. We also mention that the Dirichlet problem for the evolution of graphs in warped spaces is extensively studied in [7].

The paper is organized as follows. Section 2 describes the evolution problem in nonparametric terms. Height and boundary gradient a priori estimates for (1.2)(1.4) are presented respectively in Sections 3 and 4. Interior gradient estimates are obtained in Section 5. Some technical computations needed in the body of the proofs are compiled in an appendix. In Section 6 we prove the convergence of the mean curvature flow (1.2) for $\phi=0$ and $\mathcal{H}=0$ to slices of the form $\vartheta(\{s\} \times \Omega)$ for some $s \in \mathbb{I}$. This generalizes the corresponding convergence statement of Theorem 1.1 in 3$]$.

\section{Fundamental equations}

Since we will consider the mean curvature flow in nonparametric terms it seems adequate to describe all geometric invariants as well as their evolution equations in terms of graphical coordinates.

Let $x^{1}, \ldots, x^{n}$ be local coordinates in $P$. This system is augmented to be a coordinate system in $M$ by setting $x^{0}=s$, the flow parameter of $Y$. The tangent space of $\Sigma_{t}$ at a point $X(t, x), x \in \bar{\Omega}$, is spanned by the coordinate vector fields

$$
X_{*} \frac{\partial}{\partial x^{i}}=\vartheta_{*} \frac{\partial}{\partial x^{i}}+u_{i} \vartheta_{*} \frac{\partial}{\partial x^{0}}=\left.\frac{\partial}{\partial x^{i}}\right|_{X}+\left.u_{i} \frac{\partial}{\partial x^{0}}\right|_{X} .
$$

In terms of these coordinates the induced metric in $\Sigma_{t}$ is expressed in local components by

$$
g_{i j}=\sigma_{i j}+\frac{1}{\gamma} u_{i} u_{j}
$$

where $\gamma=\frac{1}{|Y|^{2}}$ and $\sigma_{i j}$ are the local components of the metric in $P$.

In order to compute the mean curvature of $\Sigma_{t}$, we fix $N$ as the vector field

$$
N=\frac{1}{W}\left(\gamma Y-\vartheta_{*} \nabla u\right)
$$

where $\nabla u$ is the gradient of $u$ in $P$ and

$$
W=\sqrt{\gamma+|\nabla u|^{2}} .
$$


The second fundamental form of $\Sigma_{t}$ calculated with respect to this choice of normal vector field has local components

$$
a_{i j}=\left\langle\bar{\nabla}_{X_{*} \frac{\partial}{\partial x^{i}}} X_{*} \frac{\partial}{\partial x^{j}}, N\right\rangle,
$$

where $\bar{\nabla}$ denotes the covariant derivative in $M$. We then compute

$$
\begin{aligned}
a_{i j}= & \left\langle\bar{\nabla}_{X_{*} \frac{\partial}{\partial x^{i}}} \vartheta_{*} \frac{\partial}{\partial x^{j}}, N\right\rangle+\left\langle\bar{\nabla}_{X_{*} \frac{\partial}{\partial x^{i}}} u_{j} \vartheta_{*} \frac{\partial}{\partial x^{0}}, N\right\rangle \\
= & \left\langle\bar{\nabla}_{\vartheta_{*} \frac{\partial}{\partial x^{i}}} \vartheta_{*} \frac{\partial}{\partial x^{j}}, N\right\rangle+u_{i}\left\langle\bar{\nabla}_{\vartheta_{*} \frac{\partial}{\partial x^{0}}} \vartheta_{*} \frac{\partial}{\partial x^{j}}, N\right\rangle+u_{j}\left\langle\bar{\nabla}_{\vartheta_{*} \frac{\partial}{\partial x^{i}}} \vartheta_{*} \frac{\partial}{\partial x^{0}}, N\right\rangle \\
& +u_{i, j}\left\langle\vartheta_{*} \frac{\partial}{\partial x^{0}}, N\right\rangle+u_{i} u_{j}\left\langle\bar{\nabla}_{\vartheta_{*} \frac{\partial}{\partial x^{0}}} \vartheta_{*} \frac{\partial}{\partial x^{0}}, N\right\rangle .
\end{aligned}
$$

Hence, using the fact that the maps $x \mapsto \vartheta(s, x)$ are isometries and that the hypersurfaces defined by $\{\vartheta(s, x): x \in P\}, s \in \mathbb{I}$, are totally geodesic, one concludes that

$$
\begin{aligned}
& a_{i j}=\left\langle\bar{\nabla}_{\frac{\partial}{\partial x^{i}}} \frac{\partial}{\partial x^{j}},-\frac{1}{W} \nabla u\right\rangle+u_{i}\left\langle\bar{\nabla}_{\frac{\partial}{\partial x^{j}}} Y, \frac{1}{W} \gamma Y\right\rangle+u_{j}\left\langle\bar{\nabla}_{\frac{\partial}{\partial x^{i}}} Y, \frac{1}{W} \gamma Y\right\rangle \\
& +u_{i, j}\left\langle Y, \frac{1}{W} \gamma Y\right\rangle+u_{i} u_{j}\left\langle\bar{\nabla}_{Y} Y,-\frac{1}{W} \nabla u\right\rangle .
\end{aligned}
$$

It follows from Killing's equation that

$$
a_{i j}=\frac{u_{i ; j}}{W}-\frac{u_{i}}{W} \frac{\gamma_{j}}{2 \gamma}-\frac{u_{j}}{W} \frac{\gamma_{i}}{2 \gamma}-\frac{u_{i} u_{j}}{2 W} u^{k} \frac{\gamma_{k}}{\gamma^{2}} .
$$

It turns out that $a_{i j}$ could also be expressed by

$$
a_{i j}=\frac{u_{i ; j}}{W}-\frac{u_{i}}{W} \gamma\left\langle\bar{\nabla}_{Y} Y, \frac{\partial}{\partial x^{j}}\right\rangle-\frac{u_{j}}{W} \gamma\left\langle\bar{\nabla}_{Y} Y, \frac{\partial}{\partial x^{i}}\right\rangle-\frac{u_{i} u_{j}}{W}\left\langle\bar{\nabla}_{Y} Y, \nabla u\right\rangle .
$$

Taking traces with respect to the induced metric, one obtains the following expression for the mean curvature $H$ of the hypersurface $\Sigma_{t}$ :

$$
n H=\left(\sigma^{i j}-\frac{u^{i}}{W} \frac{u^{j}}{W}\right) \frac{u_{i ; j}}{W}-\frac{2 \gamma+|\nabla u|^{2}}{W^{3}}\left\langle\frac{\bar{\nabla} \gamma}{2 \gamma}, \nabla u\right\rangle .
$$

Alternatively one has

$$
n H=\left(\sigma^{i j}-\frac{u^{i}}{W} \frac{u^{j}}{W}\right) \frac{u_{i ; j}}{W}-\frac{2 \gamma+|\nabla u|^{2}}{W^{3}} \gamma\left\langle\bar{\nabla}_{Y} Y, \nabla u\right\rangle .
$$

At this point we recall that

$$
\bar{\nabla}_{\frac{\partial}{\partial x^{i}}} Y=-\frac{1}{2} \frac{\gamma_{i}}{\gamma} Y
$$

and

$$
\bar{\nabla}_{Y} Y=\frac{1}{2} \frac{\nabla \gamma}{\gamma^{2}}
$$

which implies that

$$
\left\langle\bar{\nabla}_{Y} Y, \nabla u\right\rangle=-\left\langle\bar{\nabla}_{\nabla u} Y, Y\right\rangle=\frac{1}{2 \gamma^{2}}\langle\nabla \gamma, \nabla u\rangle .
$$

Using this one easily verifies that (2.8) may be written in divergence form as

$$
\operatorname{div} \frac{\nabla u}{W}-\frac{1}{2 \gamma W}\langle\nabla \gamma, \nabla u\rangle=n H .
$$


In fact we have

$$
\left(\frac{u^{i}}{W}\right)_{; i}=\frac{1}{W} u_{; i}^{i}-\frac{1}{W^{3}} u^{i} u^{j} u_{i ; j}-\frac{1}{2 W^{3}} u^{i} \gamma_{i} .
$$

It is worth pointing out that (2.13) is equivalent to

$$
\operatorname{div} \frac{\nabla u}{W}-\frac{\gamma}{W}\left\langle\bar{\nabla}_{Y} Y, \nabla u\right\rangle=n H .
$$

We conclude that (1.2) may be written nonparametrically as

$$
\frac{\partial u}{\partial t}=W \operatorname{div} \frac{\nabla u}{W}-W \mathcal{H}-\gamma\left\langle\bar{\nabla}_{Y} Y, \nabla u\right\rangle .
$$

Indeed it holds that

$$
n H-\mathcal{H}=\left\langle\frac{\partial X}{\partial t}, N\right\rangle=\left\langle\frac{\partial u}{\partial t} \vartheta_{*} \frac{\partial}{\partial x^{0}}, \frac{\gamma}{W} \vartheta_{*} \frac{\partial}{\partial x^{0}}\right\rangle=\frac{1}{W} \frac{\partial u}{\partial t} .
$$

Using (2.8) one verifies that (2.15) is equivalent to

$$
\frac{\partial u}{\partial t}=\left(\sigma^{i j}-\frac{u^{i}}{W} \frac{u^{j}}{W}\right) u_{i ; j}-\frac{2 \gamma+|\nabla u|^{2}}{W^{2}}\left\langle\frac{\bar{\nabla} \gamma}{2 \gamma}, \nabla u\right\rangle-W \mathcal{H} .
$$

We conclude that the Neumann problem (1.2)-(1.4) has the nonparametric form

$$
\begin{array}{ll}
u_{t}=\left(\sigma^{i j}-\frac{u^{i}}{W} \frac{u^{j}}{W}\right) u_{i ; j}-\left(\frac{1}{2 \gamma}+\frac{1}{2 W^{2}}\right) \gamma^{i} u_{i}-W \mathcal{H} & \text { in } \quad \Omega \times[0, T), \\
u(\cdot, 0)=u_{0}(\cdot) & \text { in } \quad \Omega \times\{0\},
\end{array}
$$

with boundary condition

$$
\langle N, \nu\rangle=\phi \quad \text { on } \quad \partial \Omega \times[0, T) .
$$

This boundary value problem describes the evolution of the Killing graph of the function $u(\cdot, t)$ by its mean curvature in the direction of the unit normal $N$ with prescribed contact angle at the boundary.

The standard theory for quasilinear parabolic equations [5] guarantees that the problem of solving (1.2)-(1.4) is reduced to obtaining a priori height and gradient estimates for solutions to (2.17)-(2.19).

\section{Height estimates}

From now on, we consider the parabolic linear operator given by

$$
\mathcal{L} v=g^{i j} v_{i ; j}-\left(\frac{1}{2 \gamma}+\frac{1}{2 W^{2}}\right) \gamma^{i} v_{i}-\mathcal{H} \frac{u^{i}}{W} v_{i}-v_{t},
$$

where $v \in C^{\infty}(\Omega \times[0, T))$.

Proposition 1. For a solution $u \in C^{\infty}\left(\bar{\Omega} \times\left[0, T^{*}\right]\right), T^{*}<T$, of (2.17)-(2.19), it holds that

$$
\max _{\bar{\Omega} \times\left[0, T^{*}\right]}\left|u_{t}\right|=\max _{\bar{\Omega}}\left|u_{t}(0, \cdot)\right| .
$$

Then it follows that

$$
\max _{\bar{\Omega} \times\left[0, T^{*}\right]}|u| \leq C T^{*}
$$

for a given constant $C>0$ which depends on $T^{*}$. 
Proof. First of all we verify that $u_{t}$ is a solution for a linear parabolic equation. Indeed one has

$$
\begin{aligned}
\mathcal{L} u_{t} & =g^{i j} u_{t i ; j}-\left(\frac{1}{2 \gamma}+\frac{1}{2 W^{2}}\right)\left\langle\nabla \gamma, \nabla u_{t}\right\rangle-u_{t t} \\
& =\left(g^{i j} u_{i ; j}\right)_{t}-g_{; t}^{i j} u_{i ; j}-\left(\frac{1}{2 \gamma}+\frac{1}{2 W^{2}}\right)\left\langle\nabla \gamma, \nabla u_{t}\right\rangle-u_{t t} \\
& =-g_{; t}^{i j} u_{i ; j}+\left(\frac{1}{2 \gamma}+\frac{1}{2 W^{2}}\right)_{t}\langle\nabla \gamma, \nabla u\rangle+\left(\frac{1}{2 \gamma}+\frac{1}{2 W^{2}}\right)\left\langle\nabla \gamma_{t}, \nabla u\right\rangle+W_{t} \mathcal{H} .
\end{aligned}
$$

However since $\gamma=\gamma(x)$ in (2.16) and $x$ is independent of $t$ it follows that

$$
\left(\frac{1}{2 \gamma}+\frac{1}{2 W^{2}}\right)_{t}=\left(\frac{1}{2 \gamma}\right)_{t}-\frac{1}{W^{3}} W_{t}=-\frac{1}{2 W^{4}}\left(\gamma_{t}+2 u^{k} u_{k ; t}\right)=-\frac{1}{W^{4}} u^{k} u_{t ; k} .
$$

In the same way we have

$$
W_{t}=\frac{1}{2 W}\left(\gamma_{t}+2 u^{k} u_{k ; t}\right)=\frac{1}{W} u^{k} u_{t ; k} .
$$

We conclude that

$$
\mathcal{L} u_{t}=-g_{; t}^{i j} u_{i ; j}-\frac{1}{W^{4}}\langle\nabla \gamma, \nabla u\rangle u^{k}\left(u_{t}\right)_{k}+\frac{1}{W} \mathcal{H} u^{k}\left(u_{t}\right)_{k} .
$$

Now using the fact that $\sigma_{; t}^{i j}=0$ and $\gamma_{t}=0$ we have

$$
\begin{aligned}
\mathcal{L} u_{t}= & \frac{2}{W}\left(\frac{u_{; t}^{i} u^{j}}{W}-\frac{u^{i}}{W} \frac{u^{j}}{W} W_{t}\right) u_{i ; j}-\frac{1}{W^{4}}\langle\nabla \gamma, \nabla u\rangle u^{k}\left(u_{t}\right)_{k}+\frac{1}{W} \mathcal{H} u^{k}\left(u_{t}\right)_{k} \\
= & \frac{2}{W}\left(\left(W_{i}-\frac{\gamma_{i}}{2 W}\right) u_{; t}^{i}-\left(W_{i}-\frac{\gamma_{i}}{2 W}\right) \frac{u^{i}}{W} \frac{u^{k}}{W} u_{t ; k}\right) \\
& -\frac{1}{W^{4}}\langle\nabla \gamma, \nabla u\rangle u^{k}\left(u_{t}\right)_{k}+\frac{1}{W} \mathcal{H} u^{k}\left(u_{t}\right)_{k} \\
= & \frac{2}{W}\left(W_{i}-\frac{\gamma_{i}}{2 W}\right)\left(\sigma^{i k}-\frac{u^{i}}{W} \frac{u^{k}}{W}\right) u_{t ; k}-\frac{1}{W^{4}}\langle\nabla \gamma, \nabla u\rangle u^{k}\left(u_{t}\right)_{k}+\frac{1}{W} \mathcal{H} u^{k}\left(u_{t}\right)_{k} .
\end{aligned}
$$

Hence it follows that

$$
\mathcal{L} u_{t}-\frac{2}{W} g^{i k}\left(W_{i}-\frac{\gamma_{i}}{2 W}\right)\left(u_{t}\right)_{k}+\frac{1}{W^{4}}\langle\nabla \gamma, \nabla u\rangle u^{k}\left(u_{t}\right)_{k}-\frac{1}{W} \mathcal{H} u^{k}\left(u_{t}\right)_{k}=0 .
$$

Thus fix $T^{*} \in[0, T)$ and let $\left(x_{0}, t_{0}\right)$ be a point in $\bar{\Omega} \times\left[0, T^{*}\right]$ such that

$$
u_{t}\left(x_{0}, t_{0}\right)=\max _{\bar{\Omega} \times\left[0, T^{*}\right]}\left|u_{t}\right| .
$$

Hence we choose a coordinate system adapted to the boundary $\Gamma$ in such a way that $\frac{\partial}{\partial x^{n}}=\nu$ at $x_{0}$. Then, at the point $\left(x_{0}, t_{0}\right)$ we have

$$
u_{i ; t}=u_{t ; i}=0
$$

for $1 \leq i<n$ which implies that

$$
W_{t}=\frac{1}{W} u^{n} u_{n ; t}=-\phi\left(x_{0}\right) u_{n ; t},
$$

where we used (2.19) and (3.2). On the other hand, (2.19) implies that

$$
u_{t ; n}=u_{n ; t}=-(\phi W)_{t}=-\phi\left(x_{0}\right) W_{t}
$$

at $\left(x_{0}, t_{0}\right)$. We conclude that

$$
\left(1-\phi^{2}\left(x_{0}\right)\right) u_{n ; t}=0 .
$$


However since $|\phi|<1$, it follows that $u_{t ; n}=0$ which contradicts the parabolic Hopf Lemma [5].

From this contradiction we conclude that $t_{0}=0$. Since $T^{*}$ is arbitrary, the conclusion follows.

\section{Boundary GRADIEnT ESTIMATES}

Now we will prove a gradient bound for a solution of (2.17)-2.19) by applying a modification of Korevaar's technique [4] which formerly appeared in [2].

From now on, we consider a nonnegative extension $d: \bar{\Omega} \rightarrow \mathbb{R}$ of the distance function $\operatorname{dist}_{P}(\cdot, \Gamma)$ satisfying $|\nabla d| \leq 1$ in $\bar{\Omega}$. In the same way, we consider a $C^{\infty}$ extension of the boundary data $\phi$ to the domain $\bar{\Omega}$ which we also denote by $\phi$. Then we define

$$
\eta=e^{K u} h
$$

where

$$
h=1+\alpha d-\phi\langle\nabla d, N\rangle,
$$

where $K$ and $\alpha$ are positive numbers to be fixed later.

Proposition 2. For $\alpha>0$ sufficiently large independent of $K$ and $t$, if for some $t \geq 0$ fixed, $\eta W(\cdot, t)$ attains a local maximum value at a point $x_{0} \in \partial \Omega$, then $W\left(x_{0}, t\right) \leq K$.

Proof. Let $t \geq 0$ be such that

$$
\max _{\bar{\Omega}} \eta W(t, \cdot)=\eta W\left(t, x_{0}\right)
$$

for a point $x_{0} \in \Gamma$. Hence we choose a coordinate system adapted to $\Gamma$ such that $\frac{\partial}{\partial x^{n}}=\nu$ at $x_{0}$ and

$$
u_{1}\left(x_{0}\right) \geq 0 \quad \text { and } \quad u_{i}\left(x_{0}\right)=0, \quad \text { for } \quad 2 \leq i \leq n-1 .
$$

We have at $x_{0}$

$$
0=(\eta W)_{1}=\eta_{1} W+\eta W_{1}=e^{K u}\left(W K u_{1}\left(1-\phi^{2}\right)-2 W \phi \phi_{1}+W_{1}\left(1-\phi^{2}\right)\right)
$$

from which follows that

$$
W_{1}=-K u_{1} W+\frac{2 \phi \phi_{1}}{\left(1-\phi^{2}\right)} W .
$$

On the other hand, at $x_{0}$ we have

$$
\begin{aligned}
\eta_{n}= & e^{K u}\left(K u_{n}\left(1-\phi^{2}\right)+\alpha-\phi \phi_{n}-\phi\left(\left\langle\nabla \nabla{ }_{d} N, \nabla d\right\rangle+\left\langle N, \nabla_{\nabla d} \nabla d\right\rangle\right)\right) \\
= & e^{K u}\left(K u_{n}\left(1-\phi^{2}\right)+\alpha-\phi \phi_{n}-\phi\left(\left\langle\partial_{n} \frac{1}{W}(\gamma Y-\nabla u), \partial_{n}\right\rangle\right.\right. \\
& \left.\left.+\left\langle\frac{1}{W} \nabla_{\partial_{n}}(\gamma Y-\nabla u), \partial_{n}\right\rangle\right)\right) \\
= & e^{K u}\left(K u_{n}\left(1-\phi^{2}\right)+\alpha-\phi \phi_{n}-\frac{1}{W^{2}} \phi u_{n} W_{n}+\frac{1}{W} \phi u_{n ; n}\right) .
\end{aligned}
$$

Since $(\eta W)_{n} \leq 0$ at $x_{0}$ it holds that

$$
\begin{aligned}
0 & \geq W K u_{n}\left(1-\phi^{2}\right)+\alpha W-W \phi \phi_{n}-\frac{1}{W} \phi u_{n} W_{n}+\phi u_{n ; n}+\left(1-\phi^{2}\right) W_{n} \\
& =W K u_{n}\left(1-\phi^{2}\right)+\alpha W+W_{n}+\phi u_{n ; n}+u_{n} \phi_{n} \\
& =W K u_{n}\left(1-\phi^{2}\right)+\alpha W+W_{n}+\phi u_{n ; n}-W \phi \phi_{n} .
\end{aligned}
$$


On the other hand,

$$
W_{n}=\frac{\gamma_{n}}{2 W}+\frac{1}{W}\left(u_{1} u_{1 ; n}+u_{n} u_{n ; n}\right)=\frac{\gamma_{n}}{2 W}-\frac{1}{W} \phi u_{1} W_{1}-\phi_{1} u_{1}-\phi u_{n ; n},
$$

which implies that

$$
\begin{aligned}
W_{n} & =\frac{\gamma_{n}}{2 W}-\frac{1}{W} \phi u_{1}\left(\frac{2 \phi \phi_{1} W}{1-\phi^{2}}-K u_{1} W\right)-\phi_{1} u_{1}-\phi u_{n ; n} \\
& =\frac{\gamma_{n}}{2 W}-\frac{1+\phi^{2}}{1-\phi^{2}} u_{1} \phi_{1}+K \phi u_{1}^{2}-\phi u_{n ; n} .
\end{aligned}
$$

Therefore since

$$
u_{1}^{2}=|\nabla u|^{2}-u_{n}^{2}=W^{2}-\gamma-\phi^{2} W^{2}=W^{2}\left(1-\phi^{2}\right)-\gamma,
$$

we conclude that

$$
\begin{aligned}
0 & \geq \alpha+\frac{\gamma_{n}}{2 W^{2}}-\frac{1+\phi^{2}}{1-\phi^{2}} \frac{u_{1}}{W} \phi_{1}+\frac{K \phi u_{1}^{2}}{W}-\phi \phi_{n}+K u_{n}\left(1-\phi^{2}\right) \\
& =\alpha+\frac{\gamma_{n}}{2 W^{2}}+\frac{1+\phi^{2}}{1-\phi^{2}} N_{1} \phi_{1}+K \phi\left(W\left(1-\phi^{2}\right)-\frac{\gamma}{W}\right)-\phi \phi_{n}-K \phi W\left(1-\phi^{2}\right) \\
& =\alpha+\frac{\gamma_{n}}{2 W^{2}}+\frac{1+\phi^{2}}{1-\phi^{2}} N_{1} \phi_{1}-\frac{K \phi \gamma}{W}-\phi \phi_{n} \\
& \geq \alpha+C-\frac{K \gamma}{W}
\end{aligned}
$$

for a given constant $C$ depending solely on $\gamma$ and $\phi$. It follows that $W\left(x_{0}, t\right) \leq K$ if $\alpha$ is chosen large enough and independent of $K$ and $t$.

\section{INTERIOR GRADIENT ESTIMATES}

In this section we deduce a global gradient bound using the techniques in 1 and 2]. However the more general context of warped product gives rise to a long list of additional terms which require careful tracking along the calculations.

In the sequel, we consider the parabolic linear operator given by

$$
L v=g^{i j} v_{i ; j}-\left(\frac{1}{2 \gamma}+\frac{1}{2 W^{2}}\right) \gamma^{i} v_{i}-v_{t}
$$

where $v \in C^{\infty}(\Omega \times[0, T))$.

Proposition 3. For fixed $T^{*}<T$ there exists $K>0$ sufficiently large so that if

$$
\eta W\left(x_{0}, t_{0}\right)=\max _{\bar{\Omega} \times\left[0, T^{*}\right]} \eta W
$$

for some $\left(x_{0}, t_{0}\right) \in \bar{\Omega} \times\left[0, T^{*}\right]$, then $W\left(x_{0}, t_{0}\right) \leq C$ for some constant $C$.

Proof. We can assume $x_{0} \in \Omega$ and $t_{0}>0$. At a point $\left(x_{0}, t_{0}\right)$ where $\eta W$ attains maximum value we have

$$
\eta_{i} W+\eta W_{i}=0
$$

and

$$
\frac{1}{\eta} L \eta+\frac{1}{W}\left(L W-\frac{2}{W} g^{i j} W_{i} W_{j}\right) \leq 0 .
$$


We conclude that

$$
\begin{aligned}
\frac{1}{\eta} L \eta & =K L u+\frac{1}{h} L h+K^{2} g^{i j} u_{i} u_{j}+2 K g^{i j} u_{i} \frac{h_{j}}{h} \\
& =K \mathcal{H} W+\frac{1}{h} L h+K^{2} \frac{\gamma|\nabla u|^{2}}{W^{2}}+2 K g^{i j} u_{i} \frac{h_{j}}{h}
\end{aligned}
$$

Now we have

$$
g^{i j} u_{i} h_{j}=\frac{\gamma}{W^{2}} u^{j} h_{j}=-\frac{\gamma}{W}(\alpha\langle N, \nabla d\rangle-\langle N, \nabla \phi\rangle \theta-\phi\langle N, \nabla \theta\rangle) .
$$

However

$$
\langle N, \nabla \theta\rangle=W\left\langle A Y^{T}, \nabla^{\Sigma} d\right\rangle+\left\langle\nabla_{\frac{\nabla u}{W}} \nabla d, \frac{\nabla u}{W}\right\rangle-\kappa \frac{|\nabla u|^{2}}{W^{2}} .
$$

Therefore

$$
\begin{aligned}
g^{i j} u_{i} h_{j}= & -\alpha \frac{\gamma}{W}\langle N, \nabla d\rangle+\frac{\gamma}{W}\langle N, \nabla \phi\rangle \theta+\gamma \phi\left\langle A Y^{T}, \nabla^{\Sigma} d\right\rangle \\
& +\frac{\gamma}{W} \phi\left\langle\nabla_{\frac{\nabla u}{W}} \nabla d, \frac{\nabla u}{W}\right\rangle-\gamma \phi \kappa \frac{|\nabla u|^{2}}{W^{3}} .
\end{aligned}
$$

Thus the expression for $L h$ in the Appendix allows us to conclude that

$$
\begin{aligned}
\frac{1}{\eta} L & =K \mathcal{H} W+K^{2} \frac{\gamma|\nabla u|^{2}}{W^{2}} \\
+ & \frac{2 K}{h}\left(-\alpha \frac{\gamma}{W}\langle N, \nabla d\rangle+\frac{\gamma}{W}\langle N, \nabla \phi\rangle \theta+\gamma \phi\left\langle A Y^{T}, \nabla^{\Sigma} d\right\rangle\right. \\
& \left.\quad+\frac{\gamma}{W} \phi\left\langle\nabla \frac{\nabla u}{W} \nabla d, \frac{\nabla u}{W}\right\rangle-\gamma \phi \kappa \frac{|\nabla u|^{2}}{W^{3}}\right) \\
+ & \frac{1}{h}|A|^{2} \phi \theta+n \frac{1}{h} \phi H W\left\langle A Y^{T}, \nabla^{\Sigma} d\right\rangle+\frac{1}{h}\left(\kappa \gamma-\frac{1}{2}\langle\nabla d, \nabla \gamma\rangle\right) \phi\left\langle A Y^{T}, Y^{T}\right\rangle \\
+ & \frac{2}{h}\left\langle A \nabla^{\Sigma} d, \nabla^{\Sigma} \phi\right\rangle+\frac{2}{h}\left\langle A, \nabla^{2} d\right\rangle_{\Sigma} \phi-\frac{1}{h W^{2}} \phi\left\langle A \nabla^{\Sigma} d, X_{*} \nabla \gamma\right\rangle \\
+ & \frac{1}{h}(n H-\mathcal{H})\left(\langle N, \nabla \phi\rangle \theta-\alpha \theta-\frac{1}{2 W^{2}}\langle\nabla \gamma, \nabla d\rangle \phi+\left\langle\nabla \frac{\nabla u}{W} \nabla d, \frac{\nabla u}{W}\right\rangle \phi\right)-n \frac{1}{h} \kappa H \phi \\
- & n \frac{\alpha}{h} H_{d}+\frac{1}{h}(2\langle N, \nabla \phi\rangle-\alpha)\left\langle\nabla \frac{\nabla u}{W} \nabla d, \frac{\nabla u}{W}\right\rangle+\frac{2}{h}\left\langle\nabla \frac{\nabla u}{W} \nabla d, \nabla \phi\right\rangle \\
- & \frac{1}{h} \phi\left\langle\nabla{ }_{\frac{\nabla u}{W}} \nabla d, \frac{\nabla \gamma}{2 \gamma}\right\rangle+\frac{1}{h} \phi\left\langle\nabla^{\Sigma} \mathcal{H}, \bar{\nabla} d\right\rangle+n \frac{1}{h}\left\langle\nabla H_{d}, N\right\rangle \phi-\frac{1}{h} \phi \nabla^{3} d\left(\frac{\nabla u}{W}, \frac{\nabla u}{W}, \frac{\nabla u}{W}\right) \\
+ & \frac{1}{h} \operatorname{Ric}\left(\nabla d, \frac{\nabla u}{W}\right) \phi+\frac{\gamma}{h W^{2}}\langle N, \nabla \kappa\rangle \phi-\frac{1}{h}\left(\frac{1}{2 \gamma}+\frac{1}{2 W^{2}}\right) \alpha\langle\nabla d, \nabla \gamma\rangle \\
+ & \frac{1}{h}\left(\frac{1}{2 \gamma}+\frac{1}{2 W^{2}}\right)\langle\nabla \phi, \nabla \gamma\rangle \theta \\
- & \kappa \frac{1}{h} \phi\left\langle N, \frac{\nabla \gamma}{2 \gamma}\right\rangle+\frac{2}{h} \kappa \frac{\gamma}{W^{2}}\langle\nabla \phi, N\rangle-\frac{1}{h}\left(\Delta \phi-\left\langle\nabla \frac{\nabla u}{W} \nabla \phi, \frac{\nabla u}{W}\right\rangle\right) \theta .
\end{aligned}
$$


On the other hand Lemma 3 yields

$$
\begin{aligned}
& \frac{1}{W}\left(L W-\frac{2}{W} g^{i j} W_{i} W_{j}\right)=|A|^{2}+n H W^{2}\left\langle A Y^{T}, Y^{T}\right\rangle-n H W^{2}\left\langle\frac{\nabla \gamma}{2 \gamma^{2}}, N\right\rangle \\
& -3 \frac{1}{W} \gamma\left\langle A Y^{T}, X_{*} \frac{\nabla \gamma}{2 \gamma}\right\rangle+g^{i j} \frac{\gamma_{i ; j}}{2 \gamma}-\frac{3}{4} \frac{|\nabla \gamma|^{2}}{4 \gamma^{2}}-\frac{1}{4}\left\langle\frac{\nabla \gamma}{2 \gamma}, N\right\rangle^{2}+\gamma\left\langle\bar{\nabla} \bar{\nabla}_{N} \frac{\bar{\nabla} \gamma}{2 \gamma^{2}}, N\right\rangle \\
& -\left\langle\nabla^{\Sigma} \mathcal{H}, N\right\rangle-\frac{|\nabla \gamma|^{2}}{4 \gamma} \frac{1}{W^{2}}-\frac{W_{t}}{W} .
\end{aligned}
$$

Now we use the fact that $x_{0}$ is a critical point to $\eta W$. We have

$$
e^{K u}\left(K u_{i} h+h_{i}\right) W=-e^{K u} h W_{i},
$$

which implies that

$$
-K W^{2} h N_{i} N^{i}+W h_{i} N^{i}=-h W_{i} N^{i}
$$

and then

$$
-K h|\nabla u|^{2}+W h_{i} N^{i}=-h W_{i} N^{i} .
$$

However

$$
\begin{aligned}
& W_{i} N^{i}=\frac{\gamma_{i}}{2 \gamma} N^{i} W+N_{i} N^{i} W^{3}\left\langle\frac{\nabla \gamma}{2 \gamma^{2}}, N\right\rangle+W^{2}\left\langle A Y^{T}, N^{i} X_{*} \frac{\partial}{\partial x^{i}}\right\rangle \\
& =\frac{1}{2 \gamma}\langle\nabla \gamma, N\rangle W+|\nabla u|^{2} W\left\langle\frac{\nabla \gamma}{2 \gamma^{2}}, N\right\rangle-W^{3}\left\langle A Y^{T}, Y^{T}\right\rangle
\end{aligned}
$$

and

$$
\begin{aligned}
& h_{i} N^{i}=\alpha \theta-\langle\nabla \phi, N\rangle \theta+\phi a_{i}^{j} N^{i} d_{j}-\phi\left(d_{i ; j} N^{i} N^{j}-\kappa \sigma_{i j}\right) N^{i} N^{j} \\
& =\alpha \theta-\langle\nabla \phi, N\rangle \theta-\phi W\left\langle A Y^{T}, \nabla^{\Sigma} d\right\rangle-\phi\left\langle\nabla_{\frac{\nabla u}{W}} \nabla d, \frac{\nabla u}{W}\right\rangle+\phi \kappa \frac{|\nabla u|^{2}}{W^{2}} .
\end{aligned}
$$

We then conclude that

$$
\begin{aligned}
- & K \frac{|\nabla u|^{2}}{W}+\frac{\alpha \theta}{h}-\frac{1}{h}\langle\nabla \phi, N\rangle \theta-\frac{\phi}{h} W\left\langle A Y^{T}, \nabla^{\Sigma} d\right\rangle-\frac{\phi}{h}\left\langle\nabla \frac{\nabla u}{W} \nabla d, \frac{\nabla u}{W}\right\rangle+\frac{\phi}{h} \kappa \frac{|\nabla u|^{2}}{W^{2}} \\
= & -\frac{1}{2 \gamma}\langle\nabla \gamma, N\rangle-|\nabla u|^{2}\left\langle\frac{\nabla \gamma}{2 \gamma^{2}}, N\right\rangle+W^{2}\left\langle A Y^{T}, Y^{T}\right\rangle .
\end{aligned}
$$

Moreover

$$
\begin{aligned}
-\frac{W_{t}}{W}= & \frac{\eta_{t}}{\eta}=K u_{t}+\frac{h_{t}}{h}=W K(n H-\mathcal{H})+\frac{h_{t}}{h} \\
= & n H K W-K W \mathcal{H}-\frac{1}{h}(n H-\mathcal{H})\left(\langle\nabla \phi, N\rangle \theta-\alpha \theta-\frac{\phi}{2 W^{2}}\langle\nabla \gamma, \nabla d\rangle\right) \\
& \quad+\frac{\phi}{h}\left\langle\frac{\nabla u}{W}, \nabla_{\frac{\nabla u}{W}} \nabla d\right\rangle .
\end{aligned}
$$


Then we have

$$
\begin{aligned}
& \frac{1}{W}\left(L W-\frac{2}{W} g^{i j} W_{i} W_{j}\right) \\
& =|A|^{2}+K n H \frac{\gamma}{W}+\frac{\alpha \theta}{h} n H-\frac{1}{h} n H\langle\nabla \phi, N\rangle \theta-\frac{\phi}{h} n H W\left\langle A Y^{T}, \nabla^{\Sigma} d\right\rangle \\
& -\frac{1}{h}(n H-\mathcal{H})\left(\langle\nabla \phi, N\rangle \theta-\alpha \theta-\frac{\phi}{2 W^{2}}\langle\nabla \gamma, \nabla d\rangle\right)-\frac{\phi}{h} n H\left\langle\nabla_{\frac{\nabla u}{W}} \nabla d, \frac{\nabla u}{W}\right\rangle \\
& +\frac{\phi}{h} n H \kappa \frac{|\nabla u|^{2}}{W^{2}}-3 \frac{1}{W} \gamma\left\langle A Y^{T}, X_{*} \frac{\nabla \gamma}{2 \gamma}\right\rangle+g^{i j} \frac{\gamma_{i ; j}}{2 \gamma}-\frac{3}{4} \frac{|\nabla \gamma|^{2}}{4 \gamma^{2}}-\frac{1}{4}\left\langle\frac{\nabla \gamma}{2 \gamma}, N\right\rangle^{2} \\
& +\gamma\left\langle\bar{\nabla}_{N} \frac{\bar{\nabla} \gamma}{2 \gamma^{2}}, N\right\rangle-\left\langle\nabla^{\Sigma} \mathcal{H}, N\right\rangle-\frac{|\nabla \gamma|^{2}}{4 \gamma} \frac{1}{W^{2}}-K W \mathcal{H}+\frac{\phi}{h}\left\langle\frac{\nabla u}{W}, \nabla_{\frac{\nabla u}{W}} \nabla d\right\rangle .
\end{aligned}
$$

We conclude that

$$
\frac{1}{\eta} L \eta+\frac{1}{W}\left(L W-\frac{2}{W} g^{i j} W_{i} W_{j}\right)=K^{2} \frac{\gamma|\nabla u|^{2}}{W^{2}}+\mathcal{A}+\mathcal{B},
$$

where

$$
\begin{aligned}
\mathcal{A} & =\left(1+\frac{\phi \theta}{h}\right)|A|^{2}+\frac{2 K}{h} \gamma \phi\left\langle A Y^{T}, \nabla^{\Sigma} d\right\rangle+\frac{\phi}{h}\left(\kappa \gamma-\frac{1}{2}\langle\nabla d, \nabla \gamma\rangle\right)\left\langle A Y^{T}, Y^{T}\right\rangle \\
& +\frac{2}{h}\left\langle A \nabla^{\Sigma} d, \nabla^{\Sigma} \phi\right\rangle+\frac{2}{h}\left\langle A, \nabla^{2} d\right\rangle_{\Sigma} \phi-\frac{1}{h W^{2}} \phi\left\langle A \nabla^{\Sigma} d, X_{*} \nabla \gamma\right\rangle \\
& +K n H \frac{\gamma}{W}+\frac{\alpha \theta}{h} n H-\frac{1}{h} n H\langle\nabla \phi, N\rangle \theta-\frac{\phi}{h} n H \kappa \frac{\gamma}{W^{2}}-3 \frac{1}{W} \gamma\left\langle A Y^{T}, X_{*} \frac{\nabla \gamma}{2 \gamma}\right\rangle
\end{aligned}
$$

and

$$
\begin{aligned}
& \mathcal{B}=\frac{2 K}{h}\left(-\alpha \frac{\gamma}{W}\langle N, \nabla d\rangle+\frac{\gamma}{W}\langle N, \nabla \phi\rangle \theta+\frac{\gamma}{W} \phi\left\langle\nabla_{\frac{\nabla u}{W}} \nabla d, \frac{\nabla u}{W}\right\rangle-\gamma \phi \kappa \frac{|\nabla u|^{2}}{W^{3}}\right) \\
& -\mathcal{H}\left\langle\nabla_{\frac{\nabla u}{W}} \nabla d, \frac{\nabla u}{W}\right\rangle \phi-n \frac{\alpha}{h} H_{d}+\frac{1}{h}(2\langle N, \nabla \phi\rangle-\alpha)\left\langle\nabla \frac{\nabla u}{W} \nabla d, \frac{\nabla u}{W}\right\rangle \\
& +\frac{2}{h}\left\langle\nabla_{\frac{\nabla u}{W}} \nabla d, \nabla \phi\right\rangle-\frac{1}{h} \phi\left\langle\nabla_{\frac{\nabla u}{W}} \nabla d, \frac{\nabla \gamma}{2 \gamma}\right\rangle+\frac{1}{h} \phi\left\langle\nabla^{\Sigma} \mathcal{H}, \bar{\nabla} d\right\rangle+n \frac{1}{h}\left\langle\nabla H_{d}, N\right\rangle \phi \\
& -\frac{1}{h} \phi \nabla^{3} d\left(\frac{\nabla u}{W}, \frac{\nabla u}{W}, \frac{\nabla u}{W}\right)+\frac{1}{h} \operatorname{Ric}\left(\nabla d, \frac{\nabla u}{W}\right) \phi+\frac{\gamma}{h W^{2}}\langle N, \nabla \kappa\rangle \phi \\
& -\frac{1}{h}\left(\frac{1}{2 \gamma}+\frac{1}{2 W^{2}}\right) \alpha\langle\nabla d, \nabla \gamma\rangle+\frac{1}{h}\left(\frac{1}{2 \gamma}+\frac{1}{2 W^{2}}\right)\langle\nabla \phi, \nabla \gamma\rangle \theta-\kappa \frac{1}{h} \phi\left\langle N, \frac{\nabla \gamma}{2 \gamma}\right\rangle \\
& +\frac{2}{h} \kappa \frac{\gamma}{W^{2}}\langle\nabla \phi, N\rangle-\frac{1}{h}\left(\Delta \phi-\left\langle\nabla_{\frac{\nabla u}{W}} \nabla \phi, \frac{\nabla u}{W}\right\rangle\right) \theta+g^{i j} \frac{\gamma_{i ; j}}{2 \gamma}-\frac{3}{4} \frac{|\nabla \gamma|^{2}}{4 \gamma^{2}} \\
& -\frac{1}{4}\left\langle\frac{\nabla \gamma}{2 \gamma}, N\right\rangle^{2}+\gamma\left\langle\bar{\nabla} N \frac{\bar{\nabla} \gamma}{2 \gamma^{2}}, N\right\rangle-\left\langle\nabla^{\Sigma} \mathcal{H}, N\right\rangle-\frac{|\nabla \gamma|^{2}}{4 \gamma} \frac{1}{W^{2}}+\frac{\phi}{h}\left\langle\frac{\nabla u}{W}, \nabla \frac{\nabla u}{W} \nabla d\right\rangle .
\end{aligned}
$$

However, using some standard inequalities, we obtain

$$
\begin{aligned}
\mathcal{A} & \geq\left(1+\frac{\phi \theta}{h}\right)|A|^{2}-\left(\frac{2 K \gamma}{h \sqrt{\gamma}}+\frac{\kappa}{h}+\frac{1}{2 h \gamma}|\nabla \gamma|+\frac{2}{h}|\nabla \phi|+\frac{2}{h}\left|\nabla^{2} d\right|_{\Sigma}\right. \\
& \left.+\frac{1}{h W^{2}}\left|X_{*} \nabla \gamma\right|+\frac{K \gamma \sqrt{n}}{W}+\frac{\alpha \theta \sqrt{n}}{h}+\frac{\theta \sqrt{n}}{h}|\nabla \phi|+\frac{\gamma \sqrt{n} \kappa}{h W^{2}}+\frac{3 \gamma}{\sqrt{\gamma} W}\left|X_{*} \frac{\nabla \gamma}{2 \gamma}\right|\right)|A| .
\end{aligned}
$$


Using that $W^{2} \geq \gamma$ and choosing $\alpha$ sufficiently large and depending only on $n, \gamma, \phi$ and $\kappa$, we have

$$
\begin{aligned}
& \mathcal{A} \geq \frac{1}{2}|A|^{2}-\left(\epsilon+2 \sqrt{\gamma} \frac{K}{h}+\frac{K \gamma \sqrt{n}}{W}+\frac{3 \sqrt{\gamma}}{W}\left|X_{*} \frac{\nabla \gamma}{2 \gamma}\right|\right)|A| \\
& \geq-\left(\epsilon+2 \sqrt{\gamma} \frac{K}{h}+\frac{K \gamma \sqrt{n}}{W}+\frac{3 \sqrt{\gamma}}{W}\left|X_{*} \frac{\nabla \gamma}{2 \gamma}\right|\right)^{2} .
\end{aligned}
$$

Moreover

$$
\mathcal{B} \geq-C\left(1+\frac{\alpha}{h}+\frac{\alpha}{h W^{2}}+\frac{1}{h}+\frac{1}{W^{2}}+\frac{1}{h W^{2}}+K \frac{\alpha}{h}+\frac{K}{h}\right),
$$

where $C$ is a constant depending on $n, \gamma, \phi, d, \kappa$ and $\mathcal{H}$.

Hence we obtain

$$
\begin{aligned}
& \frac{1}{\eta} L \eta+\frac{1}{W}\left(\mathcal{L} W-\frac{2}{W} g^{i j} W_{i} W_{j}\right) \geq K^{2} \frac{\gamma|\nabla u|^{2}}{W^{2}}-C(\epsilon)-\frac{K}{W} C(\epsilon, \gamma, n)-\frac{K^{2}}{W^{2}} C(\gamma, n) \\
& -\frac{1}{W} C(\gamma, \epsilon)-\frac{K}{W^{2}} C(\gamma, n)-\frac{K^{2}}{h^{2}} C(\gamma)-\frac{K^{2}}{h W} C(\gamma, n)-\frac{1}{W^{2}} C(\gamma)-\frac{K}{h W} C(\gamma) \\
& -K \frac{\alpha}{h} C-\frac{K}{h} C(\epsilon, \gamma)-C-\frac{\alpha}{h} C-\frac{\alpha}{h W^{2}} C-\frac{1}{h} C-\frac{1}{W^{2}} C-\frac{1}{h W^{2}} C .
\end{aligned}
$$

Then

$$
\begin{gathered}
-K^{2} \frac{\gamma|\nabla u|^{2}}{W^{2}} \geq-C\left(\frac{K^{2}}{W^{2}}+\frac{K}{W^{2}}+\frac{1}{W}+\frac{K}{h W}+\frac{K^{2}}{h^{2}}+\frac{\alpha}{h W^{2}}+\frac{1}{W^{2}}+\frac{1}{h W^{2}}\right. \\
\left.+\frac{K}{W}+\frac{1}{W}+K \frac{\alpha}{h}+\frac{K}{h}+\frac{\alpha}{h}+\frac{1}{h}+1\right) .
\end{gathered}
$$

It follows that

$$
\begin{aligned}
& \left(K^{2} \gamma-\left(\frac{K^{2}}{h^{2}}+K \frac{\alpha}{h}+\frac{K}{h}+\frac{1+\alpha}{h}+1\right) C\right) W^{2} \leq\left(K^{2}+K+\frac{1+\alpha}{h}+1\right) C \\
& +\left(K+\frac{K}{h}+1\right) C W .
\end{aligned}
$$

Now suppose that $W\left(x_{0}, t_{0}\right) \geq 1$. Otherwise we are done. In this case we have $W \leq W^{2}$ and absorbing the terms with $W$ into the one with $W^{2}$ transforms the inequality above into

$$
\begin{aligned}
& \left(K^{2} \gamma-\frac{K^{2}}{h^{2}} C-\frac{K}{h} C-K C-C-\frac{1}{h}(\alpha+1)(K+1) C\right) W^{2} \\
& \leq\left(K^{2}+K+1+\frac{1}{h}(\alpha+1)\right) C .
\end{aligned}
$$

If $d_{0}=d\left(x_{0}\right)$, then choosing $\alpha \geq 1 /\left(C\left(d_{0}\right) d_{0}-1\right)$ for some constant $C\left(d_{0}\right)>1 / d_{0}$, we obtain $(1+\alpha) / h \leq C\left(d_{0}\right)$ which implies that

$$
\left(K^{2} \gamma-\frac{K^{2}}{h^{2}} C-\frac{K}{h} C-K C\left(d_{0}\right)-C\left(d_{0}\right)\right) W^{2} \leq\left(K^{2}+K+C\left(d_{0}\right)\right) C .
$$

Then for $\alpha>\frac{1}{d_{0}} \max \{1, \sqrt{2 C / \gamma}\}$ we have

$$
\left(K^{2} \frac{\gamma}{2}-K C\left(d_{0}\right)-C\left(d_{0}\right)\right) W^{2} \leq\left(K^{2}+K+C\left(d_{0}\right)\right) C .
$$


It follows that for $K>\frac{C\left(d_{0}\right)+\sqrt{C\left(d_{0}\right)^{2}+2 \gamma C\left(d_{0}\right)}}{\gamma}$ we have $K^{2} \frac{\gamma}{2}-K C\left(d_{0}\right)-C\left(d_{0}\right)>0$ and

$$
W^{2} \leq \frac{C\left(K^{2}+K+C\left(d_{0}\right)\right)}{K^{2} \frac{\gamma}{2}-K C\left(d_{0}\right)-C\left(d_{0}\right)} .
$$

This finishes the proof of the proposition.

Theorem 2. There exists a unique solution $u: \bar{\Omega} \times[0, \infty) \rightarrow \mathbb{I}$ to the problem (1.2)-(1.4) .

Proof. Propositions 1, 2 and 3 yield the global gradient bound

$$
W(x, t) \leq W\left(x_{0}, t_{0}\right) \frac{\eta(x, t)}{\eta\left(x_{0}, t_{0}\right)} \leq C_{1} e^{C_{2} M T^{*}}
$$

for $(x, t) \in \bar{\Omega} \times\left[0, T^{*}\right]$, where $C_{1}$ and $C_{2}$ are positive constants and

$$
M=\max _{\bar{\Omega} \times\left[0, T^{*}\right]}\left|u-u_{0}\right| .
$$

It results that (2.17) is uniformly parabolic and then the standard theory of quasilinear parabolic PDEs may be applied to assure the existence of a unique smooth solution to (2.17)-(2.19).

\section{Asymptotic Behavior}

Suppose from now on that $\mathcal{H}=0$ and $\phi=0$. In the particular case when the evolving functions have the form $u(x, t)=v(x)+C t,(x, t) \in \bar{\Omega} \times[0, T)$, the initial value problem (2.17)-(2.19) becomes

$$
\begin{array}{lr}
\operatorname{div} \frac{\nabla v}{W}-\gamma\left\langle\bar{\nabla}_{Y} Y, \frac{\nabla v}{W}\right\rangle=\frac{C}{W} & \text { in } \quad \Omega, \\
\langle\nu, N\rangle=0 \quad \text { on } \quad \partial \Omega .
\end{array}
$$

Conversely, notice that if $v(x)$ is a solution of (6.1)-(6.2), then $u=v+C t$ is a solution of (2.17) which is translating along the flow lines of $Y$ with speed $C$.

Now observe that

$\operatorname{div} \frac{\nabla v}{W}-\gamma\left\langle\bar{\nabla}_{Y} Y, \frac{\nabla v}{W}\right\rangle=\operatorname{div} \frac{\nabla v}{W}+\gamma\left\langle\bar{\nabla}_{\frac{\nabla v}{W}} Y, Y\right\rangle=\operatorname{div} \frac{\nabla v}{W}+\gamma\left\langle\bar{\nabla}_{Y} \frac{\nabla v}{W} Y, Y\right\rangle=\operatorname{div}_{M} \frac{\nabla v}{W}$.

Therefore it follows from the divergence theorem that

$$
\int_{\vartheta([0, s] \times \bar{\Omega})} \frac{C}{W}+\mathcal{H}=-\int_{\vartheta([0, s] \times \Gamma)}\left\langle\frac{\nabla v}{W}, \nu\right\rangle=\int_{\vartheta([0, s] \times \Gamma)}\langle N, \nu\rangle=\int_{\vartheta([0, s] \times \Gamma)} \phi .
$$

Since the integrands do not depend on $s$ we have

$$
\int_{\Omega} C \frac{1}{\sqrt{\gamma} W}=\int_{\Gamma} \frac{1}{\sqrt{\gamma}} \phi
$$

from which results that

$$
C=0 .
$$

We then obtain the following height estimate.

Proposition 4. Given a solution $u(x, t)$ of (2.17) there exists a constant $M$ such that

$$
|u(x, t)| \leq M
$$

for $(x, t) \in \bar{\Omega} \times[0,+\infty)$. 
Proof. We observe that since $C$ is necessarily zero, $v=$ cte is a solution to (6.1). In particular the constant functions $v_{1}=\inf _{\Omega} u_{0}$ and $v_{2}=\sup _{\Omega} u_{0}$ are solutions of (6.1) with $v_{1} \leq u_{0} \leq v_{2}$. Hence the parabolic maximum principle implies that

$$
v_{1} \leq u(\cdot, t) \leq v_{2}
$$

for $t \in[0, T)$ from which we obtain (6.6).

Now, proceeding as in [3], we prove the following convergence result.

Theorem 3. Suppose that $\mathcal{H}=0$ and $\phi=0$. Then $\lim _{t \rightarrow \infty} u_{t}=0$. In particular the mean curvature flow converges to a slice of the form $\vartheta(\{s\} \times \bar{\Omega})$ for some $s \in \mathbb{I}$.

Proof. It is immediate that $v=s$ is a trivial solution to (6.1) with (necessarily) $C=0$. We also have

$$
\frac{d}{d t} \int_{\Omega} W=\int_{\Omega} \frac{u^{i} u_{i ; t}}{W}=-\int_{\Omega} \frac{u_{t}^{2}}{W}-\int_{\Omega} \frac{1}{2 W^{3}}\langle\nabla u, \nabla \gamma\rangle-\int_{\Omega} \frac{|\nabla u|^{2}}{2 \gamma W^{2}}\langle\nabla u, \nabla \gamma\rangle .
$$

Therefore

$$
-\int_{\Omega} \frac{u_{t}^{2}}{W}=\frac{d}{d t}\left(\int_{\Omega} W\right)+\int_{\Omega} \frac{1}{2 W^{3}}\langle\nabla u, \nabla \gamma\rangle+\int_{\Omega} \frac{|\nabla u|^{2}}{2 \gamma W^{2}}\langle\nabla u, \nabla \gamma\rangle
$$

It follows that

$$
\begin{aligned}
& \int_{0}^{T} \int_{\Omega} \frac{u_{t}^{2}}{W}=-\int_{\Omega} W(x, T)+\int_{\Omega} W(x, 0) \\
& +\int_{0}^{T} \int_{\Omega} \frac{1}{2 W^{3}}\langle\nabla u, \nabla \gamma\rangle+\int_{0}^{T} \int_{\Omega} \frac{|\nabla u|^{2}}{2 \gamma W^{2}}\langle\nabla u, \nabla \gamma\rangle \leq \widetilde{C}
\end{aligned}
$$

for some positive constant $\widetilde{C}$. It also follows that $\lim _{t \rightarrow \infty} \frac{u_{t}^{2}}{W}=0$. Since $W$ is bounded, then $\lim _{t \rightarrow \infty} u_{t}=0$. This finishes the proof of the theorem.

\section{Appendix}

In what follows, $I I$ and $A$ denote respectively the second fundamental form and the Weingarten map of $\Sigma_{t}$. Their components are given by

$$
a_{i j}=I I\left(X_{*} \frac{\partial}{\partial x^{i}}, X_{*} \frac{\partial}{\partial x^{j}}\right):=\left\langle A X_{*} \frac{\partial}{\partial x^{i}}, X_{*} \frac{\partial}{\partial x^{j}}\right\rangle .
$$

Some lemmata will be needed in the sequel. Their content could also be of independent interest for other applications.

Lemma 1. Denote $\theta=\langle\nabla d, N\rangle$. The differentials of the functions $\theta$ and $h$ have components given by

$$
\theta_{i}=-a_{i}^{j} d_{j}+\left(d_{i ; j}-\kappa \sigma_{i j}\right) N^{j}
$$

and

$$
h_{i}=\left(\alpha \delta_{i}^{j}+\phi a_{i}^{j}\right) d_{j}-\left(\phi\left(d_{i ; j}-\kappa \sigma_{i j}\right)+\phi_{i} d_{j}\right) N^{j},
$$

respectively, where $\kappa=\left\langle\gamma \bar{\nabla}_{Y} Y, \nabla d\right\rangle$. 
Proof. We have

$$
\begin{aligned}
& \frac{\partial \theta}{\partial x^{i}}=X_{*} \frac{\partial}{\partial x^{i}}\langle N, \bar{\nabla} d\rangle=\left\langle\bar{\nabla}_{X_{*} \frac{\partial}{\partial x^{i}}} N, \bar{\nabla} d\right\rangle+\left\langle N, \bar{\nabla}_{X_{*} \frac{\partial}{\partial x^{i}}} \bar{\nabla} d\right\rangle \\
& =-\left\langle A X_{*} \frac{\partial}{\partial x^{i}}, \bar{\nabla} d\right\rangle+\left\langle N, \bar{\nabla} \frac{\partial}{\partial x^{i}}+u_{i} \frac{\partial}{\partial x^{0}} \bar{\nabla} d\right\rangle \\
& =-\left\langle A X_{*} \frac{\partial}{\partial x^{i}}, \bar{\nabla} d\right\rangle+\frac{\gamma}{W}\left\langle\frac{\partial}{\partial x^{0}}, \bar{\nabla} \frac{\partial}{\partial x^{i}} \bar{\nabla} d\right\rangle-\left\langle\frac{\nabla u}{W}, \bar{\nabla} \frac{\partial}{\partial x^{i}} \bar{\nabla} d\right\rangle \\
& +u_{i} \frac{\gamma}{W}\left\langle\frac{\partial}{\partial x^{0}}, \bar{\nabla} \frac{\partial}{\partial x^{0}} \bar{\nabla} d\right\rangle-u_{i}\left\langle\frac{\nabla u}{W}, \bar{\nabla} \frac{\partial}{\partial x^{0}} \bar{\nabla} d\right\rangle .
\end{aligned}
$$

Since $P$ is totally geodesic we have

$$
\left\langle\frac{\partial}{\partial x^{0}}, \bar{\nabla} \frac{\partial}{\partial x^{i}} \bar{\nabla} d\right\rangle=\left\langle\frac{\partial}{\partial x^{0}}, \bar{\nabla} \frac{\partial}{\partial x^{i}} \nabla d\right\rangle=0 .
$$

Moreover we compute

$$
\left\langle\frac{\partial}{\partial x^{0}}, \bar{\nabla} \frac{\partial}{\partial x^{0}} \bar{\nabla} d\right\rangle=|Y|^{2}\left\langle\frac{Y}{|Y|}, \bar{\nabla} \frac{Y}{|Y|} \bar{\nabla} d\right\rangle=|Y|^{2} \kappa=\frac{1}{\gamma} \kappa
$$

and

$$
\left\langle\frac{\nabla u}{W}, \bar{\nabla} \frac{\partial}{\partial x^{0}} \bar{\nabla} d\right\rangle=\left\langle\frac{\nabla u}{W}, \bar{\nabla}_{\bar{\nabla} d} \frac{\partial}{\partial x^{0}}\right\rangle+\left\langle\frac{\nabla u}{W},\left[\frac{\partial}{\partial x^{0}}, \bar{\nabla} d\right]\right\rangle=0,
$$

where we used the fact that $\left[\frac{\partial}{\partial x^{0}}, \bar{\nabla} d\right]=0$ and that $P$ is totally geodesic.

Thus we conclude that

However

$$
\frac{\partial \theta}{\partial x^{i}}=-\left\langle A X_{*} \frac{\partial}{\partial x^{i}}, \bar{\nabla} d\right\rangle-\left\langle\frac{\nabla u}{W}, \nabla \frac{\partial}{\partial x^{i}} \nabla d\right\rangle+\kappa \frac{u_{i}}{W} .
$$

$$
\left\langle A X_{*} \frac{\partial}{\partial x^{i}}, \bar{\nabla} d\right\rangle=a_{i}^{j}\left\langle X_{*} \frac{\partial}{\partial x^{j}}, \bar{\nabla} d\right\rangle=a_{i}^{j}\left\langle\frac{\partial}{\partial x^{j}}+u_{j} Y, \bar{\nabla} d\right\rangle=a_{i}^{j} d_{j}=g^{j k} a_{i k} d_{j} .
$$

Therefore we write

$$
\theta_{i}=-g^{j k} a_{i k} d_{j}+\left(d_{i ; j}-\kappa \sigma_{i j}\right) N^{j}
$$

This finishes the proof of the proposition.

We denote the components of the tensor $X^{*} I I$ in $P$ by

$$
b_{i j}=X^{*} I I\left(\frac{\partial}{\partial x^{i}}, \frac{\partial}{\partial x^{j}}\right):=\left\langle A X_{*} \frac{\partial}{\partial x^{i}}, X_{*} \frac{\partial}{\partial x^{j}}\right\rangle .
$$

Notice that the covariant derivatives of $X^{*} I I$ and $I I$ are related by

$$
\begin{aligned}
& \nabla_{k} b_{i j}=\left\langle\left(\nabla_{X_{*}}^{\Sigma} \frac{\partial}{\partial x^{k}} A\right) X_{*} \frac{\partial}{\partial x^{i}}, X_{*} \frac{\partial}{\partial x^{j}}\right\rangle+\left\langle A X_{*} \frac{\partial}{\partial x^{j}}, \bar{\nabla}_{X_{*} \frac{\partial}{\partial x^{k}}} X_{*} \frac{\partial}{\partial x^{i}}-X_{*} \nabla_{\frac{\partial}{\partial x^{k}}} \frac{\partial}{\partial x^{i}}\right\rangle \\
& +\left\langle A X_{*} \frac{\partial}{\partial x^{i}}, \bar{\nabla}_{X_{*} \frac{\partial}{\partial x^{k}}} X_{*} \frac{\partial}{\partial x^{j}}-X_{*} \nabla_{\frac{\partial}{\partial x^{k}}} \frac{\partial}{\partial x^{j}}\right\rangle .
\end{aligned}
$$

However since $X_{*} \frac{\partial}{\partial x^{i}}=\frac{\partial}{\partial x^{i}}+u_{i} Y$ we compute

Therefore

$$
\begin{gathered}
\bar{\nabla}_{X_{*} \frac{\partial}{\partial x^{k}}} X_{*} \frac{\partial}{\partial x^{i}}-X_{*} \nabla_{\frac{\partial}{\partial x^{k}}} \frac{\partial}{\partial x^{i}}=\bar{\nabla}_{\frac{\partial}{\partial x^{k}}} \frac{\partial}{\partial x^{i}}+u_{i, k} Y+u_{i} \bar{\nabla}_{\frac{\partial}{\partial x^{k}}} Y \\
+u_{k} \bar{\nabla}_{Y} \frac{\partial}{\partial x^{i}}+u_{i} u_{k} \bar{\nabla}_{Y} Y-\nabla_{\frac{\partial}{\partial x^{k}}} \frac{\partial}{\partial x^{i}}-\left\langle\nabla u, \nabla_{\frac{\partial}{\partial x^{k}}} \frac{\partial}{\partial x^{i}}\right\rangle Y .
\end{gathered}
$$

$$
\bar{\nabla}_{X_{*} \frac{\partial}{\partial x^{k}}} X_{*} \frac{\partial}{\partial x^{i}}-X_{*} \nabla_{\frac{\partial}{\partial x^{k}}} \frac{\partial}{\partial x^{i}}=u_{i ; k} Y+u_{i} \overline{\nabla_{\frac{\partial}{\partial x^{k}}}} Y+u_{k} \bar{\nabla}_{\frac{\partial}{\partial x^{i}}} Y+u_{i} u_{k} \bar{\nabla}_{Y} Y
$$


Hence using (2.6), (2.10) and (2.11) we obtain

$$
\begin{aligned}
& \bar{\nabla}_{X_{*} \frac{\partial}{\partial x^{k}}} X_{*} \frac{\partial}{\partial x^{i}}-X_{*} \nabla_{\frac{\partial}{\partial x^{k}}} \frac{\partial}{\partial x^{i}}=\left(W a_{i k}+u_{i} u_{k} u^{l} \frac{\gamma_{l}}{2 \gamma^{2}}\right) Y+\frac{1}{2} u_{i} u_{k} \frac{\nabla \gamma}{\gamma^{2}} \\
& =W a_{i k} Y+\frac{1}{2 \gamma^{2}} u_{i} u_{k}(\langle\nabla u, \nabla \gamma\rangle Y+\nabla \gamma)=W a_{i k} Y+\frac{1}{2 \gamma^{2}} u_{i} u_{k} X_{*} \nabla \gamma .
\end{aligned}
$$

Hence it follows that

$$
\begin{aligned}
& \left\langle A X_{*} \frac{\partial}{\partial x^{j}}, \bar{\nabla}_{X_{*} \frac{\partial}{\partial x^{k}}} X_{*} \frac{\partial}{\partial x^{i}}-X_{*} \nabla_{\frac{\partial}{\partial x^{k}}} \frac{\partial}{\partial x^{i}}\right\rangle=\left\langle A X_{*} \frac{\partial}{\partial x^{j}}, \frac{u_{i} u_{k}}{2 \gamma^{2}} X_{*} \nabla \gamma+W a_{i k} Y\right\rangle \\
& =\frac{1}{\gamma} W a_{i k} a_{j}^{l} u_{l}+\frac{u_{i} u_{k}}{2 \gamma^{2}} a_{j l} \gamma^{l} .
\end{aligned}
$$

We conclude that

$$
\begin{aligned}
& \nabla_{k} b_{i j}=\left\langle\left(\nabla_{X_{*} \frac{\partial}{\partial x^{k}}}^{\Sigma} A\right) X_{*} \frac{\partial}{\partial x^{i}}, X_{*} \frac{\partial}{\partial x^{j}}\right\rangle+\frac{1}{\gamma} W a_{i k} a_{j}^{l} u_{l}+\frac{u_{i} u_{k}}{2 \gamma^{2}} a_{j l} \gamma^{l} \\
& +\frac{1}{\gamma} W a_{j k} a_{i}^{l} u_{l}+\frac{u_{j} u_{k}}{2 \gamma^{2}} a_{i l} \gamma^{l},
\end{aligned}
$$

that is,

$$
\nabla_{k} b_{i j}=\nabla_{k}^{\Sigma} a_{i j}+\frac{1}{\gamma} W a_{i k} a_{j}^{l} u_{l}+\frac{1}{\gamma} W a_{j k} a_{i}^{l} u_{l}+\frac{u_{i} u_{k}}{2 \gamma^{2}} a_{j l} \gamma^{l}+\frac{u_{j} u_{k}}{2 \gamma^{2}} a_{i l} \gamma^{l} .
$$

Now we use (7.6) for computing the Hessian of the function $\theta$.

Lemma 2. The trace of the Hessian of $\theta$ in $\Omega$ calculated with respect to the metric in $\Sigma$ is given by

$$
\begin{aligned}
& g^{i k} \theta_{i ; k}=-|A|^{2} \theta-2\left\langle\nabla^{2} d, X^{*} I I\right\rangle_{\Sigma}-n\left\langle\nabla^{\Sigma} H, \nabla^{\Sigma} d\right\rangle-n H W\left\langle A Y^{T}, \nabla^{\Sigma} d\right\rangle \\
& -\operatorname{Ric}\left(\nabla d, \frac{\nabla u}{W}\right)-\operatorname{tr}_{\Sigma} \nabla \frac{\nabla u}{W} \nabla^{2} d-\frac{|\nabla u|^{2}}{W^{2}}\left\langle A \nabla^{\Sigma} d, X_{*} \frac{\nabla \gamma}{2 \gamma}\right\rangle+\frac{1}{2}\left\langle A Y^{T}, Y^{T}\right\rangle\langle\nabla d, \nabla \gamma\rangle \\
& -\frac{1}{2 W^{2}} \nabla^{2} d\left(\frac{\nabla u}{W}, \nabla \gamma\right)-\frac{\gamma}{W^{2}}\langle N, \nabla \kappa\rangle+\kappa\left(n H-\gamma\left\langle A Y^{T}, Y^{T}\right\rangle\right)-\kappa \frac{1}{2 W^{2}}\langle N, \nabla \gamma\rangle .
\end{aligned}
$$

Proof. Notice that we may write (7.4) as

$$
\theta_{i}=-g^{j l} b_{i l} d_{j}+\left(d_{i ; j}-\kappa \sigma_{i j}\right) N^{j} .
$$

Hence we have

$$
\begin{aligned}
& n g^{i k} \theta_{i ; k}=-g^{i k}\left(g^{j l} b_{i l} d_{j}\right)_{; k}+g^{i k}\left(d_{i ; j k}-\kappa_{k} \sigma_{i j}\right) N^{j}+g^{i k}\left(d_{i ; j}-\kappa \sigma_{i j}\right) N_{; k}^{j} \\
& =-g^{i k}\left(g^{j l} b_{i l} d_{j}\right)_{; k}+g^{i k}\left(d_{i ; k j}+R_{j k i}^{l} d_{l}-\kappa_{k} \sigma_{i j}\right) N^{j}-g^{i k}\left(d_{i ; j}-\kappa \sigma_{i j}\right)\left(a_{k}^{j}-N_{k} \frac{\gamma^{j}}{2 \gamma}\right) .
\end{aligned}
$$

However

$$
\begin{aligned}
& g^{i k}\left(g^{j l} b_{i l} d_{j}\right)_{; k}=g^{j l} g^{i k} b_{i l ; k} d_{j}+g^{i k} g_{; k}^{j l} b_{i l} d_{j}+g^{i k} g^{j l} b_{i l} d_{j ; k} \\
& \quad=g^{j l} g^{i k}\left(\nabla_{k}^{\Sigma} a_{i l}+\frac{1}{\gamma} W a_{i k} a_{l}^{m} u_{m}+\frac{1}{\gamma} W a_{l k} a_{i}^{m} u_{m}+u_{i} u_{k} a_{l m} \frac{\gamma^{m}}{2 \gamma^{2}}+u_{l} u_{k} a_{i m} \frac{\gamma^{m}}{2 \gamma^{2}}\right) d_{j} \\
& \quad+g^{i k} g_{; k}^{j l} b_{i l} d_{j}+g^{i k} g^{j l} b_{i l} d_{j ; k} .
\end{aligned}
$$


Hence using Codazzi's equation we obtain

$$
\begin{aligned}
& g^{i k}\left(g^{j l} b_{i l} d_{j}\right)_{; k}=g^{j l}\left(n H_{l}+n \frac{1}{\gamma} W H a_{l}^{m} u_{m}+\frac{1}{\gamma} W a_{l}^{i} a_{i}^{m} u_{m}+\frac{|\nabla u|^{2}}{W^{2}} a_{l m} \frac{\gamma^{m}}{2 \gamma}\right. \\
& \left.\quad+u_{l} u_{k} a_{m}^{k} \frac{\gamma^{m}}{2 \gamma^{2}}\right) d_{j}+g^{j l} g^{i k}\left\langle\bar{R}\left(X_{*} \frac{\partial}{\partial x^{i}}, X_{*} \frac{\partial}{\partial x^{k}}\right) N, X_{*} \frac{\partial}{\partial x^{l}}\right\rangle d_{j}+g^{i k} g_{; k}^{j l} b_{i l} d_{j} \\
& \quad+g^{i k} g^{j l} b_{i l} d_{j ; k} .
\end{aligned}
$$

Using that $g^{j l} u_{l}=\frac{\gamma}{W^{2}} u^{j}$ we conclude that

$$
\begin{aligned}
& g^{i k}\left(g^{j l} b_{i l} d_{j}\right)_{; k}=n g^{j l} H_{l} d_{j}-n \frac{1}{\gamma} W^{2} H g^{j l} a_{l}^{m} N_{m} d_{j}-\frac{1}{\gamma} W^{2} g^{j l} a_{l}^{i} a_{i}^{m} N_{m} d_{j} \\
& +\frac{|\nabla u|^{2}}{W^{2}} a_{m}^{j} \frac{\gamma^{m}}{2 \gamma} d_{j}+N^{j} N_{k} a_{m}^{k} \frac{\gamma^{m}}{2 \gamma} d_{j}+g^{i k} g_{; k}^{j l} b_{i l} d_{j}+g^{i k} g^{j l} b_{i l} d_{j ; k} .
\end{aligned}
$$

However we have

$g_{; k}^{j l}=\left(\sigma^{j l}-N^{j} N^{l}\right)_{; k}=-N_{; k}^{j} N^{l}-N^{j} N_{; k}^{l}=\left(a_{k}^{j}-N_{k} \frac{\gamma^{j}}{2 \gamma}\right) N^{l}+N^{j}\left(a_{k}^{l}-N_{k} \frac{\gamma^{l}}{2 \gamma}\right)$

and

$$
\begin{aligned}
& \bar{\nabla}_{\frac{\partial}{\partial x^{k}}} N=\bar{\nabla}_{X_{*} \frac{\partial}{\partial x^{k}}} N-\bar{\nabla}_{u_{k} Y} N=-A X_{*} \frac{\partial}{\partial x^{k}}-u_{k} \bar{\nabla}_{Y}\left(\frac{\gamma}{W} Y-\frac{\nabla u}{W}\right) \\
& =-A X_{*} \frac{\partial}{\partial x^{k}}-\frac{u_{k}}{2 W}\left(\frac{\nabla \gamma}{\gamma}+\left\langle\nabla u, \frac{\nabla \gamma}{\gamma}\right\rangle Y\right),
\end{aligned}
$$

from which follows that

$$
\begin{aligned}
& g^{i k}\left(g^{j l} b_{i l} d_{j}\right)_{; k}=n g^{j l} H_{l} d_{j}-n \frac{1}{\gamma} W^{2} H a_{l}^{m} N_{m} g^{j l} d_{j}-\frac{1}{\gamma} W^{2} a_{l}^{i} a_{i}^{m} N_{m} g^{j l} d_{j} \\
& +\frac{|\nabla u|^{2}}{W^{2}} a_{m}^{j} \frac{\gamma^{m}}{2 \gamma} d_{j}+N^{j} N_{k} a_{m}^{k} \frac{\gamma^{m}}{2 \gamma} d_{j}+a_{l}^{k} a_{k}^{j} N^{l} d_{j} \\
& -a_{l}^{k} N_{k} N^{l} \frac{\gamma^{j}}{2 \gamma} d_{j}+a_{l}^{k} a_{k}^{l} N^{j} d_{j}-a_{l}^{k} N_{k} \frac{\gamma^{l}}{2 \gamma} N^{j} d_{j}+g^{j l} a_{l}^{k} d_{j ; k} .
\end{aligned}
$$

Therefore

$$
\begin{aligned}
& g^{i k} \theta_{i ; k}=-n g^{j l} H_{l} d_{j}+n \frac{1}{\gamma} W^{2} H a_{l}^{m} N_{m} g^{j l} d_{j}+\frac{1}{\gamma} W^{2} a_{l}^{i} a_{i}^{m} N_{m} g^{j l} d_{j} \\
& -\frac{|\nabla u|^{2}}{W^{2}} a_{m}^{j} \frac{\gamma^{m}}{2 \gamma} d_{j}-a_{l}^{k} a_{k}^{j} N^{l} d_{j}+a_{l}^{k} N_{k} N^{l}\left\langle\nabla d, \frac{\nabla \gamma}{2 \gamma}\right\rangle-a_{l}^{k} a_{k}^{l} \theta-g^{j l} a_{l}^{k} d_{j ; k} \\
& +g^{i k}\left(d_{i ; k j}+R_{j k i}^{l} d_{l}-\kappa_{k} \sigma_{i j}\right) N^{j}-g^{i k}\left(d_{i ; j}-\kappa \sigma_{i j}\right)\left(a_{k}^{j}-N_{k} \frac{\gamma^{j}}{2 \gamma}\right) .
\end{aligned}
$$

Now using the fact that $g^{i j} u_{j}=\frac{\gamma}{W^{2}} u^{i}$ and therefore $g^{i j} N_{j}=\frac{\gamma}{W^{2}} N^{i}$ we obtain

$$
\begin{aligned}
& a_{i}^{m} N_{m}=g^{k m} a_{i k} N_{m}=\frac{\gamma}{W^{2}} a_{i k} N^{k}=\frac{\gamma}{W^{2}}\left\langle A X_{*} \frac{\partial}{\partial x^{i}}, N^{k} X_{*} \frac{\partial}{\partial x^{k}}\right\rangle \\
& =\frac{\gamma}{W^{2}}\left\langle A X_{*} \frac{\partial}{\partial x^{i}}, N^{k} \frac{\partial}{\partial x^{k}}+\left\langle N^{k} \frac{\partial}{\partial x^{k}}, \nabla u\right\rangle Y\right\rangle \\
& =\frac{\gamma}{W^{2}}\left\langle A X_{*} \frac{\partial}{\partial x^{i}}, N-\frac{\gamma}{W} Y+\langle N, \nabla u\rangle Y\right\rangle \\
& =-\frac{\gamma}{W^{2}}\left\langle A X_{*} \frac{\partial}{\partial x^{i}}, Y\right\rangle\left(\frac{\gamma}{W}+\frac{|\nabla u|^{2}}{W}\right)=-\frac{\gamma}{W}\left\langle A X_{*} \frac{\partial}{\partial x^{i}}, Y\right\rangle=-\frac{\gamma}{W}\left\langle A Y^{T}, X_{*} \frac{\partial}{\partial x^{i}}\right\rangle .
\end{aligned}
$$


Therefore

$$
a_{l}^{m} N_{m} g^{j l} d_{j}=-\frac{\gamma}{W}\left\langle A Y^{T}, g^{j l} d_{j} X_{*} \frac{\partial}{\partial x^{l}}\right\rangle=-\frac{\gamma}{W}\left\langle A Y^{T}, \nabla^{\Sigma} d\right\rangle .
$$

Moreover notice that

$$
a_{l}^{k} N^{l}=g^{k m} a_{m l} N^{l}=-g^{k m} W\left\langle A Y^{T}, X_{*} \frac{\partial}{\partial x^{m}}\right\rangle
$$

and

Similarly we have

$$
a_{i k} N^{k}=-W\left\langle A Y^{T}, X_{*} \frac{\partial}{\partial x^{i}}\right\rangle
$$

$$
a_{k}^{j} d_{j}=g^{j m} d_{j}\left\langle A X_{*} \frac{\partial}{\partial x^{k}}, X_{*} \frac{\partial}{\partial x^{m}}\right\rangle=\left\langle A X_{*} \frac{\partial}{\partial x^{k}}, \nabla^{\Sigma} d\right\rangle=\left\langle A \nabla^{\Sigma} d, X_{*} \frac{\partial}{\partial x^{k}}\right\rangle .
$$

Replacing the above we obtain

$$
\begin{aligned}
& g^{i k} \theta_{i ; k}=-n\left\langle\nabla^{\Sigma} H, \nabla^{\Sigma} d\right\rangle-n H W\left\langle A Y^{T}, \nabla^{\Sigma} d\right\rangle-W\left\langle A Y^{T}, A \nabla^{\Sigma} d\right\rangle \\
& -\frac{|\nabla u|^{2}}{W^{2}}\left\langle A \nabla^{\Sigma} d, X_{*} \frac{\nabla \gamma}{2 \gamma}\right\rangle \\
& +W\left\langle A Y^{T}, A \nabla^{\Sigma} d\right\rangle+\gamma\left\langle A Y^{T}, Y^{T}\right\rangle\left\langle\nabla d, \frac{\nabla \gamma}{2 \gamma}\right\rangle-|A|^{2} \theta-g^{j l} a_{l}^{k} d_{j ; k} \\
& +g^{i k}\left(d_{i ; k j}+R_{j k i}^{l} d_{l}-\kappa_{k} \sigma_{i j}\right) N^{j}-g^{i k}\left(d_{i ; j}-\kappa \sigma_{i j}\right)\left(a_{k}^{j}-N_{k} \frac{\gamma^{j}}{2 \gamma}\right) .
\end{aligned}
$$

Therefore

$$
\begin{aligned}
& g^{i k} \theta_{i ; k}=-n\left\langle\nabla^{\Sigma} H, \nabla^{\Sigma} d\right\rangle-n H W\left\langle A Y^{T}, \nabla^{\Sigma} d\right\rangle-\frac{|\nabla u|^{2}}{W^{2}}\left\langle A \nabla^{\Sigma} d, X_{*} \frac{\nabla \gamma}{2 \gamma}\right\rangle \\
& +\gamma\left\langle A Y^{T}, Y^{T}\right\rangle\left\langle\nabla d, \frac{\nabla \gamma}{2 \gamma}\right\rangle-|A|^{2} \theta-g^{j l} a_{l}^{k} d_{j ; k} \\
& \quad+g^{i k}\left(d_{i ; k j}+R_{j k i}^{l} d_{l}-\kappa_{k} \sigma_{i j}\right) N^{j}-g^{i k}\left(d_{i ; j}-\kappa \sigma_{i j}\right)\left(a_{k}^{j}-N_{k} \frac{\gamma^{j}}{2 \gamma}\right) .
\end{aligned}
$$

However

$$
g^{i k} \sigma_{i j}=g^{i k}\left(g_{i j}-\frac{u_{i} u_{j}}{\gamma}\right)=\delta_{j}^{k}-\frac{1}{W^{2}} u^{k} u_{j}=\delta_{j}^{k}-N^{k} N_{j} .
$$

Hence we have

$$
\begin{aligned}
& g^{i k} \theta_{i ; k}=-n\left\langle\nabla^{\Sigma} H, \nabla^{\Sigma} d\right\rangle-n H W\left\langle A Y^{T}, \nabla^{\Sigma} d\right\rangle-\frac{|\nabla u|^{2}}{W^{2}}\left\langle A \nabla^{\Sigma} d, X_{*} \frac{\nabla \gamma}{2 \gamma}\right\rangle \\
& +\frac{1}{2}\left\langle A Y^{T}, Y^{T}\right\rangle\langle\nabla d, \nabla \gamma\rangle-|A|^{2} \theta-2 g^{i k} g^{j l} d_{i ; j} a_{k l}+\frac{1}{2 W^{2}} d_{i ; j} N^{i} \gamma^{j} \\
& +g^{i k} d_{i ; k j} N^{j}-\operatorname{Ric}\left(\nabla d, \frac{\nabla u}{W}\right)-\frac{\gamma}{W^{2}}\langle N, \nabla \kappa\rangle+\kappa\left(n H-\gamma\left\langle A Y^{T}, Y^{T}\right\rangle\right) \\
& -\kappa \frac{1}{2 W^{2}}\langle N, \nabla \gamma\rangle .
\end{aligned}
$$

This finishes the proof of the lemma.

Using Lemma 2 we will obtain an expression for $L h$. Notice that

$$
h_{i ; k}=\alpha d_{i ; k}-\phi_{i} \theta_{k}-\phi_{k} \theta_{i}-\phi_{i ; k} \theta-\phi \theta_{i ; k} .
$$


Moreover it holds that

$$
\begin{aligned}
& 2 g^{i k} \phi_{i} \theta_{k}=2 g^{i k} \phi_{i}\left\langle A \nabla^{\Sigma} d, X_{*} \frac{\partial}{\partial x^{k}}\right\rangle-2 g^{i k} d_{k ; l} \phi_{i} N^{l}+2 \kappa g^{i k} \sigma_{k l} \phi_{i} N^{l} \\
& =2\left\langle A \nabla^{\Sigma} d, \nabla^{\Sigma} \phi\right\rangle-2 g^{i k} d_{k ; l} \phi_{i} N^{l}+2 \kappa \frac{\gamma}{W^{2}}\langle\nabla \phi, N\rangle .
\end{aligned}
$$

We conclude that

$$
\begin{aligned}
& g^{i k} h_{i ; k}=\alpha g^{i k} d_{i ; k}+2\left\langle A \nabla^{\Sigma} d, \nabla^{\Sigma} \phi\right\rangle-2 g^{i k} d_{k ; l} \phi_{i} N^{l}+2 \kappa \frac{\gamma}{W^{2}}\langle\nabla \phi, N\rangle-g^{i k} \phi_{i ; k} \theta \\
& +n \phi\left\langle\nabla^{\Sigma} H, \nabla^{\Sigma} d\right\rangle+n \phi H W\left\langle A Y^{T}, \nabla^{\Sigma} d\right\rangle+\frac{|\nabla u|^{2}}{W^{2}} \phi\left\langle A \nabla^{\Sigma} d, X_{*} \frac{\nabla \gamma}{2 \gamma}\right\rangle \\
& \quad-\frac{1}{2} \phi\left\langle A Y^{T}, Y^{T}\right\rangle\langle\nabla d, \nabla \gamma\rangle+|A|^{2} \phi \theta+2 g^{i k} g^{j l} d_{i ; j} a_{k l} \phi-\frac{1}{2 W^{2}} \phi d_{i ; j} N^{i} \gamma^{j} \\
& -g^{i k} d_{i ; k j} N^{j} \phi+\operatorname{Ric}\left(\nabla d, \frac{\nabla u}{W}\right) \phi+\frac{\gamma}{W^{2}}\langle N, \nabla \kappa\rangle \phi-\kappa\left(n H-\gamma\left\langle A Y^{T}, Y^{T}\right\rangle\right) \phi \\
& +\kappa \frac{1}{2 W^{2}}\langle N, \nabla \gamma\rangle \phi .
\end{aligned}
$$

Now we compute the derivatives with respect to $t$. We have

$$
\begin{gathered}
\theta_{t}=X_{*} \frac{\partial}{\partial t}\langle N, \bar{\nabla} d\rangle=\left\langle\bar{\nabla}_{X_{*} \frac{\partial}{\partial t}} N, \bar{\nabla} d\right\rangle+\left\langle N, \bar{\nabla}_{X_{*} \frac{\partial}{\partial t}} \bar{\nabla} d\right\rangle \\
=-\left\langle\nabla^{\Sigma}(n H-\mathcal{H}), \bar{\nabla} d\right\rangle+(n H-\mathcal{H})\left\langle N, \bar{\nabla}_{N} \bar{\nabla} d\right\rangle .
\end{gathered}
$$

However

$$
\left\langle N, \bar{\nabla}_{N} \bar{\nabla} d\right\rangle=-\frac{1}{2 W^{2}}\langle\bar{\nabla} \gamma, \bar{\nabla} d\rangle+\left\langle\frac{\nabla u}{W}, \bar{\nabla}_{\frac{\nabla u}{W}} \bar{\nabla} d\right\rangle
$$

Hence we have

$$
\theta_{t}=-\left\langle\nabla^{\Sigma}(n H-\mathcal{H}), \bar{\nabla} d\right\rangle+(n H-\mathcal{H})\left(-\frac{1}{2 W^{2}}\langle\nabla \gamma, \nabla d\rangle+\left\langle\frac{\nabla u}{W}, \bar{\nabla}_{\frac{\nabla u}{W}} \bar{\nabla} d\right\rangle\right) .
$$

Moreover we have

$$
d_{t}=\left\langle X_{*} \frac{\partial}{\partial t}, \bar{\nabla} d\right\rangle=(n H-\mathcal{H})\langle N, \bar{\nabla} d\rangle=(n H-\mathcal{H}) \theta
$$

Therefore

$$
\begin{aligned}
& h_{t}=\alpha(n H-\mathcal{H}) \theta-(n H-\mathcal{H})\langle N, \bar{\nabla} \phi\rangle \theta+\phi\left\langle\nabla^{\Sigma}(n H-\mathcal{H}), \bar{\nabla} d\right\rangle \\
& -\phi(n H-\mathcal{H})\left(-\frac{1}{2 W^{2}}\langle\nabla \gamma, \nabla d\rangle+\left\langle\frac{\nabla u}{W}, \bar{\nabla}_{\frac{\nabla u}{W}} \bar{\nabla} d\right\rangle\right) .
\end{aligned}
$$

We also compute

$$
\langle\nabla \gamma, \nabla h\rangle=\alpha\langle\nabla d, \nabla \gamma\rangle+\phi\left\langle A \nabla^{\Sigma} d, X_{*} \nabla \gamma\right\rangle-\langle\nabla \phi, \nabla \gamma\rangle \theta-\phi d_{i ; j} \gamma^{i} N^{j}+\kappa \phi\langle N, \nabla \gamma\rangle .
$$

Now we obtain

and

$$
g^{i k} d_{i ; k}=\Delta d-\left\langle\nabla_{\frac{\nabla u}{W}} \nabla d, \frac{\nabla u}{W}\right\rangle=-n H_{d}-\left\langle\nabla_{\frac{\nabla u}{W}} \nabla d, \frac{\nabla u}{W}\right\rangle
$$

$$
\begin{aligned}
g^{i k} d_{k ; l} \phi_{i} N^{l} & =d_{k ; l} \phi^{k} N^{l}-d_{k ; l} N^{k} N^{l} N^{i} \phi_{i} \\
& =-\left\langle\nabla_{\frac{\nabla u}{W}} \nabla d, \nabla \phi\right\rangle-\left\langle\nabla_{\frac{\nabla u}{W}} \nabla d, \frac{\nabla u}{W}\right\rangle\langle N, \nabla \phi\rangle .
\end{aligned}
$$

Moreover we have

$$
g^{i j} \phi_{i ; j}=\Delta \phi-\left\langle\nabla_{\frac{\nabla u}{W}} \nabla \phi, \frac{\nabla u}{W}\right\rangle
$$


and

$$
\begin{aligned}
g^{i k} d_{i ; k j} N^{j} & =\left(\sigma^{i k} d_{i ; k}\right)_{; j} N^{j}-d_{i ; k j} N^{i} N^{k} N^{j} \\
& =-n\left(H_{d}\right)_{j} N^{j}+\nabla^{3} d\left(\frac{\nabla u}{W}, \frac{\nabla u}{W}, \frac{\nabla u}{W}\right) .
\end{aligned}
$$

Therefore grouping and rearranging these expressions we obtain

$$
\begin{aligned}
& L h=|A|^{2} \phi \theta+n \phi H W\left\langle A Y^{T}, \nabla^{\Sigma} d\right\rangle+\left(\kappa \gamma-\frac{1}{2}\langle\nabla d, \nabla \gamma\rangle\right) \phi\left\langle A Y^{T}, Y^{T}\right\rangle \\
& +2\left\langle A \nabla^{\Sigma} d, \nabla^{\Sigma} \phi\right\rangle+2\left\langle A, \nabla^{2} d\right\rangle_{\Sigma} \phi-\frac{1}{W^{2}} \phi\left\langle A \nabla^{\Sigma} d, X_{*} \nabla \gamma\right\rangle \\
& (n H-\mathcal{H})\left(\langle N, \nabla \phi\rangle-\alpha \theta-\frac{1}{2 W^{2}}\langle\nabla \gamma, \nabla d\rangle \phi+\left\langle\nabla_{\frac{\nabla u}{W}} \nabla d, \frac{\nabla u}{W}\right\rangle \phi\right)-n \kappa H \phi \\
& -n \alpha H_{d}+(2\langle N, \nabla \phi\rangle-\alpha)\left\langle\nabla_{\frac{\nabla u}{W}} \nabla d, \frac{\nabla u}{W}\right\rangle+2\left\langle\nabla_{\frac{\nabla u}{W}} \nabla d, \nabla \phi\right\rangle \\
& -\phi\left\langle\nabla_{\frac{\nabla u}{W}} \nabla d, \frac{\nabla \gamma}{2 \gamma}\right\rangle+\phi\left\langle\nabla^{\Sigma} \mathcal{H}, \bar{\nabla} d\right\rangle+n\left\langle\nabla H_{d}, N\right\rangle \phi \\
& -\phi \nabla^{3} d\left(\frac{\nabla u}{W}, \frac{\nabla u}{W}, \frac{\nabla u}{W}\right)+\operatorname{Ric}\left(\nabla d, \frac{\nabla u}{W}\right) \phi \\
& +\frac{\gamma}{W^{2}}\langle N, \nabla \kappa\rangle \phi-\left(\frac{1}{2 \gamma}+\frac{1}{2 W^{2}}\right) \alpha\langle\nabla d, \nabla \gamma\rangle+\left(\frac{1}{2 \gamma}+\frac{1}{2 W^{2}}\right)\langle\nabla \phi, \nabla \gamma\rangle \theta \\
& -\kappa \phi\left\langle N, \frac{\nabla \gamma}{2 \gamma}\right\rangle+2 \kappa \frac{\gamma}{W^{2}}\langle\nabla \phi, N\rangle-\left(\Delta \phi-\left\langle\nabla_{\frac{\nabla u}{W}} \nabla \phi, \frac{\nabla u}{W}\right\rangle\right) \theta .
\end{aligned}
$$

Lemma 3. We have

$$
\begin{aligned}
& L W-\frac{2}{W} g^{i j} W_{i} W_{j} \\
& =|A|^{2} W+n H W^{3}\left\langle A Y^{T}, Y^{T}\right\rangle-n H W^{3}\left\langle\frac{\nabla \gamma}{2 \gamma^{2}}, N\right\rangle-3 \gamma\left\langle A Y^{T}, X_{*} \frac{\nabla \gamma}{2 \gamma}\right\rangle \\
& \quad+g^{i j} \frac{\gamma_{i ; j}}{2 \gamma} W-\frac{3}{4} \frac{|\nabla \gamma|^{2}}{4 \gamma^{2}} W-\frac{1}{4}\left\langle\frac{\nabla \gamma}{2 \gamma}, N\right\rangle^{2} W+\gamma W\left\langle\bar{\nabla}_{N} \frac{\bar{\nabla} \gamma}{2 \gamma^{2}}, N\right\rangle-W\left\langle\nabla^{\Sigma} \mathcal{H}, N\right\rangle \\
& -\frac{|\nabla \gamma|^{2}}{4 \gamma} \frac{1}{W}-W_{t} .
\end{aligned}
$$

Proof. Notice that

$$
\begin{aligned}
& W_{i}=-W^{2}\left(\left\langle\bar{\nabla}_{X_{*} \frac{\partial}{\partial x^{i}}} Y, N\right\rangle+\left\langle Y, \bar{\nabla}_{X_{*} \frac{\partial}{\partial x^{i}}} N\right\rangle\right) \\
& =-W^{2}\left(\left\langle\bar{\nabla}_{\frac{\partial}{\partial x^{i}}} Y, N\right\rangle+u_{i}\left\langle\bar{\nabla}_{Y} Y, N\right\rangle-\left\langle Y, A X_{*} \frac{\partial}{\partial x^{i}}\right\rangle\right) \\
& =-W^{2}\left(-\frac{\gamma_{i}}{2 \gamma}\langle Y, N\rangle+u_{i}\left\langle\frac{\nabla \gamma}{2 \gamma^{2}}, N\right\rangle-\left\langle Y, A X_{*} \frac{\partial}{\partial x^{i}}\right\rangle\right) .
\end{aligned}
$$

Therefore

$$
W_{i}=\frac{\gamma_{i}}{2 \gamma} W+N_{i} W^{3}\left\langle\frac{\nabla \gamma}{2 \gamma^{2}}, N\right\rangle+W^{2}\left\langle A Y^{T}, X_{*} \frac{\partial}{\partial x^{i}}\right\rangle
$$

However

$$
\left\langle A Y^{T}, X_{*} \frac{\partial}{\partial x^{i}}\right\rangle=g^{k l}\left\langle Y, X_{*} \frac{\partial}{\partial x^{k}}\right\rangle\left\langle X_{*} \frac{\partial}{\partial x^{l}}, A X_{*} \frac{\partial}{\partial x^{i}}\right\rangle=g^{k l}\left\langle Y, u_{k} Y\right\rangle b_{i l}=\frac{1}{W^{2}} u^{l} b_{i l} .
$$


Hence it follows that

$$
W_{i}=\frac{\gamma_{i}}{2 \gamma} W+N_{i} W^{3}\left\langle\frac{\nabla \gamma}{2 \gamma^{2}}, N\right\rangle-W N^{l} b_{i l}
$$

Hence we obtain

$$
\begin{aligned}
& \frac{1}{W} g^{i j} W_{i} W_{j}=\frac{\left|\nabla^{\Sigma} \gamma\right|^{2}}{4 \gamma^{2}} W+W\langle\nabla \gamma, N\rangle\left\langle\frac{\nabla \gamma}{2 \gamma^{2}}, N\right\rangle+\left\langle A Y^{T}, \frac{\nabla^{\Sigma} \gamma}{\gamma}\right\rangle W^{2} \\
& +\gamma|\nabla u|^{2}\left\langle\frac{\nabla \gamma}{2 \gamma^{2}}, N\right\rangle^{2} W-\left\langle A Y^{T}, Y^{T}\right\rangle\left\langle\frac{\nabla \gamma}{\gamma}, N\right\rangle W^{3}+\left\langle A Y^{T}, A Y^{T}\right\rangle W^{3} .
\end{aligned}
$$

Now we compute

$$
\begin{aligned}
& W_{i ; j}=\left(\frac{\gamma_{i ; j}}{2 \gamma}-\frac{\gamma_{i} \gamma_{j}}{2 \gamma^{2}}\right) W+\frac{\gamma_{i}}{2 \gamma} W_{j}+N_{i ; j} W^{3}\left\langle\frac{\nabla \gamma}{2 \gamma^{2}}, N\right\rangle+3 N_{i} W^{2} W_{j}\left\langle\frac{\nabla \gamma}{2 \gamma^{2}}, N\right\rangle \\
& +N_{i} W^{3}\left(\left\langle\bar{\nabla}_{X_{*} \frac{\partial}{\partial x^{j}}} \frac{\bar{\nabla} \gamma}{2 \gamma^{2}}, N\right\rangle-\left\langle\frac{\nabla \gamma}{2 \gamma^{2}}, A X_{*} \frac{\partial}{\partial x^{j}}\right\rangle\right)-W_{j} N^{l} b_{i l}-W N_{; j}^{l} b_{i l}-W N^{l} b_{i l ; j} .
\end{aligned}
$$

However we have

$$
g^{i j} \frac{\gamma_{i}}{2 \gamma} W_{j}=\frac{\left|\nabla^{\Sigma} \gamma\right|^{2}}{4 \gamma^{2}} W+\left\langle\frac{\nabla \gamma}{2 \gamma}, N\right\rangle^{2} W+W^{2}\left\langle A Y^{T}, \frac{\nabla^{\Sigma} \gamma}{2 \gamma}\right\rangle
$$

and

$$
\begin{aligned}
& g^{i j} N_{i ; j}=g^{i j} \sigma_{i k} N_{; j}^{k}=-\left(\delta_{k}^{j}-N^{j} N_{k}\right)\left(a_{j}^{k}-N_{j} \frac{\gamma^{k}}{2 \gamma}\right) \\
& =-n H+\frac{\gamma}{W^{2}}\left\langle N, \frac{\nabla \gamma}{2 \gamma}\right\rangle+\gamma\left\langle A Y^{T}, Y^{T}\right\rangle .
\end{aligned}
$$

Moreover we compute

$$
g^{i j} N_{i} W_{j}=\frac{\gamma}{W}\left\langle N, \frac{\nabla \gamma}{2 \gamma}\right\rangle+\frac{\gamma|\nabla u|^{2}}{W}\left\langle\frac{\nabla \gamma}{2 \gamma^{2}}, N\right\rangle-\gamma W\left\langle A Y^{T}, Y^{T}\right\rangle
$$

and

$$
\begin{aligned}
& g^{i j} N_{i} W^{3}\left(\left\langle\bar{\nabla}_{X_{*} \frac{\partial}{\partial x^{j}}} \frac{\bar{\nabla} \gamma}{2 \gamma^{2}}, N\right\rangle-\left\langle\frac{\bar{\nabla} \gamma}{2 \gamma^{2}}, A X_{*} \frac{\partial}{\partial x^{j}}\right\rangle\right) \\
& =\gamma W\left(\left\langle\bar{\nabla}_{N-W Y} \frac{\bar{\nabla} \gamma}{2 \gamma^{2}}, N\right\rangle-\left\langle A \frac{\nabla^{\Sigma} \gamma}{2 \gamma^{2}},-W Y\right\rangle\right) \\
& =\gamma W\left\langle\bar{\nabla}_{N} \frac{\bar{\nabla} \gamma}{2 \gamma^{2}}, N\right\rangle+W \frac{|\nabla \gamma|^{2}}{4 \gamma^{2}}+\gamma W^{2}\left\langle A \frac{\nabla^{\Sigma} \gamma}{2 \gamma^{2}}, Y^{T}\right\rangle .
\end{aligned}
$$

We also have

$$
\begin{aligned}
2 W g^{i j} W_{j}\left\langle A Y^{T}, X_{*} \frac{\partial}{\partial x^{i}}\right\rangle= & 2 W^{2}\left\langle A Y^{T}, \frac{\nabla^{\Sigma} \gamma}{2 \gamma}\right\rangle-W^{3}\left\langle\frac{\nabla \gamma}{\gamma}, N\right\rangle\left\langle A Y^{T}, Y^{T}\right\rangle \\
& +2 W^{3}\left\langle A Y^{T}, A Y^{T}\right\rangle .
\end{aligned}
$$

Now we compute

$$
\begin{aligned}
& g^{i j} W N^{l} b_{i l ; j}=W N^{l} g^{i j} \nabla_{j}^{\Sigma} a_{i l}+\frac{1}{\gamma} W^{2} g^{i j} a_{i j} a_{l}^{m} N^{l} u_{m}+\frac{1}{\gamma} W^{2} g^{i j} a_{l j} N^{l} a_{i}^{m} u_{m} \\
& +W g^{i j} \frac{u_{i} u_{j}}{2 \gamma^{2}} a_{l m} N^{l} \gamma^{m}+W g^{i j} N^{l} \frac{u_{l} u_{j}}{2 \gamma^{2}} a_{i m} \gamma^{m} .
\end{aligned}
$$


Hence we have

$$
\begin{aligned}
& g^{i j} W N^{l} b_{i l ; j}=W N^{l}\left(n \nabla_{l}^{\Sigma} H+g^{i j}\left\langle\bar{R}\left(X_{*} \frac{\partial}{\partial x^{i}}, X_{*} \frac{\partial}{\partial x^{j}}\right) N, X_{*} \frac{\partial}{\partial x^{l}}\right\rangle\right) \\
& +n H W^{2}\left\langle A Y^{T}, N^{k} X_{*} \frac{\partial}{\partial x^{k}}\right\rangle \\
& +W^{2} g^{i j}\left\langle A Y^{T}, X_{*} \frac{\partial}{\partial x^{j}}\right\rangle\left(-W\left\langle A Y^{T}, X_{*} \frac{\partial}{\partial x^{i}}\right\rangle\right) \\
& -|\nabla u|^{2}\left\langle A Y^{T}, X_{*} \frac{\nabla \gamma}{2 \gamma}\right\rangle+\frac{|\nabla u|^{2}}{2 \gamma W}\left(-W\left\langle A Y^{T}, X_{*} \frac{\partial}{\partial x^{m}}\right\rangle\right) \gamma^{m} .
\end{aligned}
$$

Therefore

$$
\begin{aligned}
g^{i j} W N^{l} b_{i l ; j}= & n W N^{l} \nabla_{l}^{\Sigma} H-n H W^{3}\left\langle A Y^{T}, Y^{T}\right\rangle-W^{3}\left\langle A Y^{T}, A Y^{T}\right\rangle \\
& -|\nabla u|^{2}\left\langle A Y^{T}, X_{*} \frac{\nabla \gamma}{\gamma}\right\rangle .
\end{aligned}
$$

Moreover

$$
g^{i j} W_{j} N^{l} b_{i l}=-W^{2}\left\langle A Y^{T}, \frac{\nabla^{\Sigma} \gamma}{2 \gamma}\right\rangle+W^{3}\left\langle\frac{\nabla \gamma}{2 \gamma}, N\right\rangle\left\langle A Y^{T}, Y^{T}\right\rangle-W^{3}\left\langle A Y^{T}, A Y^{T}\right\rangle
$$

and

$$
W g^{i j} N_{; j}^{l} b_{i l}=-W g^{i j}\left(a_{j}^{l}-N_{j} \frac{\gamma^{l}}{2 \gamma}\right) a_{i l}=-|A|^{2} W-\frac{1}{2}\left\langle A Y^{T}, X_{*} \nabla \gamma\right\rangle
$$

We conclude that

$$
\begin{aligned}
& g^{i j} W_{i ; j}=|A|^{2} W+2 W^{3}\left\langle A Y^{T}, A Y^{T}\right\rangle+\left(n H-3\left\langle\frac{\nabla \gamma}{2 \gamma}, N\right\rangle\right) W^{3}\left\langle A Y^{T}, Y^{T}\right\rangle \\
& +3 W^{2}\left\langle A Y^{T}, \frac{\nabla^{\Sigma} \gamma}{2 \gamma}\right\rangle+|\nabla u|^{2}\left\langle A Y^{T}, X_{*} \frac{\nabla \gamma}{\gamma}\right\rangle+\frac{1}{2}\left\langle A Y^{T}, X_{*} \nabla \gamma\right\rangle \\
& +g^{i j} \frac{\gamma_{i ; j}}{2 \gamma} W-\frac{\left|\nabla^{\Sigma} \gamma\right|^{2}}{4 \gamma^{2}} W+\frac{|\nabla \gamma|^{2}}{4 \gamma^{2}} W+\left(5 W+3 \frac{W}{\gamma}|\nabla u|^{2}\right)\left\langle\frac{\nabla \gamma}{2 \gamma}, N\right\rangle^{2} \\
& -n H W^{3}\left\langle\frac{\nabla \gamma}{2 \gamma^{2}}, N\right\rangle+\gamma W\left\langle\bar{\nabla}_{N} \frac{\bar{\nabla} \gamma}{2 \gamma^{2}}, N\right\rangle-n W N^{l} \nabla_{l}^{\Sigma} H .
\end{aligned}
$$

Now

$$
\langle\nabla \gamma, \nabla W\rangle=\frac{|\nabla \gamma|^{2}}{2 \gamma} W+\frac{1}{2 \gamma^{2}}\langle\nabla \gamma, N\rangle^{2} W^{3}+W^{2}\left\langle A Y^{T}, X_{*} \nabla \gamma\right\rangle
$$


Hence

$$
\begin{aligned}
& L W-\frac{2}{W} g^{i j} W_{i} W_{j}=|A|^{2} W+\left(n H+\left\langle\frac{\nabla \gamma}{2 \gamma}, N\right\rangle\right) W^{3}\left\langle A Y^{T}, Y^{T}\right\rangle \\
& -W^{2}\left\langle A Y^{T}, \frac{\nabla^{\Sigma} \gamma}{2 \gamma}\right\rangle+|\nabla u|^{2}\left\langle A Y^{T}, X_{*} \frac{\nabla \gamma}{\gamma}\right\rangle+\frac{1}{2}\left\langle A Y^{T}, X_{*} \nabla \gamma\right\rangle \\
& -\left(\frac{1}{2 \gamma}+\frac{1}{2 W^{2}}\right) W^{2}\left\langle A Y^{T}, X_{*} \nabla \gamma\right\rangle \\
& +g^{i j} \frac{\gamma_{i ; j}}{2 \gamma} W-\frac{3}{4} \frac{\left|\nabla^{\Sigma} \gamma\right|^{2}}{4 \gamma^{2}} W+\frac{|\nabla \gamma|^{2}}{4 \gamma^{2}} W+\left(5 W+3 \frac{W}{\gamma}|\nabla u|^{2}\right)\left\langle\frac{\nabla \gamma}{2 \gamma}, N\right\rangle^{2} \\
& -n H W^{3}\left\langle\frac{\nabla \gamma}{2 \gamma^{2}}, N\right\rangle+\gamma W\left\langle\bar{\nabla}_{N} \frac{\bar{\nabla} \gamma}{2 \gamma^{2}}, N\right\rangle-n W N^{l} \nabla_{l}^{\Sigma} H \\
& -\left(\frac{1}{2 \gamma}+\frac{1}{2 W^{2}}\right)\left(\frac{|\nabla \gamma|^{2}}{2 \gamma} W+\frac{1}{2 \gamma^{2}}\langle\nabla \gamma, N\rangle^{2} W^{3}\right)-\frac{1}{\gamma^{2}}\langle\nabla \gamma, N\rangle^{2} W \\
& -2 \gamma|\nabla u|^{2}\left\langle\frac{\nabla \gamma}{2 \gamma^{2}}, N\right\rangle^{2} W-W_{t} .
\end{aligned}
$$

However

$$
\begin{gathered}
3 \frac{W}{\gamma}|\nabla u|^{2}\left\langle\frac{\nabla \gamma}{2 \gamma}, N\right\rangle^{2}-2 \gamma|\nabla u|^{2}\left\langle\frac{\nabla \gamma}{2 \gamma^{2}}, N\right\rangle^{2} W=\frac{W}{\gamma}|\nabla u|^{2}\left\langle\frac{\nabla \gamma}{2 \gamma}, N\right\rangle^{2}, \\
5 W\left\langle\frac{\nabla \gamma}{2 \gamma}, N\right\rangle^{2}-\frac{1}{\gamma^{2}}\langle\nabla \gamma, N\rangle^{2} W=\left\langle\frac{\nabla \gamma}{2 \gamma}, N\right\rangle^{2} W
\end{gathered}
$$

and

$$
\begin{aligned}
& -W^{2}\left\langle A Y^{T}, \frac{\nabla^{\Sigma} \gamma}{2 \gamma}\right\rangle+|\nabla u|^{2}\left\langle A Y^{T}, X_{*} \frac{\nabla \gamma}{\gamma}\right\rangle+\frac{1}{2}\left\langle A Y^{T}, X_{*} \nabla \gamma\right\rangle \\
& -\left(\frac{1}{2 \gamma}+\frac{1}{2 W^{2}}\right) W^{2}\left\langle A Y^{T}, X_{*} \nabla \gamma\right\rangle \\
& =-3 \gamma\left\langle A Y^{T}, X_{*} \frac{\nabla \gamma}{2 \gamma}\right\rangle-W^{3}\left\langle\frac{\nabla \gamma}{2 \gamma}, N\right\rangle\left\langle A Y^{T}, Y^{T}\right\rangle .
\end{aligned}
$$

Moreover we compute

$$
\begin{aligned}
& \left(\frac{1}{2 \gamma}+\frac{1}{2 W^{2}}\right)\left(\frac{|\nabla \gamma|^{2}}{2 \gamma} W+\frac{1}{2 \gamma^{2}}\langle\nabla \gamma, N\rangle^{2} W^{3}\right) \\
& =\frac{|\nabla \gamma|^{2}}{4 \gamma^{2}} W+\frac{1}{\gamma} W^{3}\left\langle\frac{\nabla \gamma}{2 \gamma}, N\right\rangle^{2}+\frac{|\nabla \gamma|^{2}}{4 \gamma} \frac{1}{W}+\left\langle\frac{\nabla \gamma}{2 \gamma}, N\right\rangle^{2} W
\end{aligned}
$$

and

$$
-n W N^{l} \nabla_{l}^{\Sigma} H=-n W\left\langle\nabla^{\Sigma} H, N\right\rangle=-W\left\langle\nabla^{\Sigma} \mathcal{H}, N\right\rangle .
$$


We conclude that

$$
\begin{aligned}
& L W-\frac{2}{W} g^{i j} W_{i} W_{j}=|A|^{2} W+n H W^{3}\left\langle A Y^{T}, Y^{T}\right\rangle-3 \gamma\left\langle A Y^{T}, X_{*} \frac{\nabla \gamma}{2 \gamma}\right\rangle \\
& +g^{i j} \frac{\gamma_{i ; j}}{2 \gamma} W-\frac{3}{4} \frac{\left|\nabla^{\Sigma} \gamma\right|^{2}}{4 \gamma^{2}} W+\frac{|\nabla u|^{2}}{\gamma}\left\langle\frac{\nabla \gamma}{2 \gamma}, N\right\rangle^{2} W \\
& -n H W^{3}\left\langle\frac{\nabla \gamma}{2 \gamma^{2}}, N\right\rangle+\gamma W\left\langle\bar{\nabla}_{N} \frac{\bar{\nabla} \gamma}{2 \gamma^{2}}, N\right\rangle-W\left\langle\nabla^{\Sigma} \mathcal{H}, N\right\rangle \\
& -\frac{1}{\gamma} W^{3}\left\langle\frac{\nabla \gamma}{2 \gamma}, N\right\rangle^{2}-\frac{|\nabla \gamma|^{2}}{4 \gamma} \frac{1}{W}-W_{t} .
\end{aligned}
$$

However

$$
\frac{|\nabla u|^{2}}{\gamma}\left\langle\frac{\nabla \gamma}{2 \gamma}, N\right\rangle^{2} W-\frac{1}{\gamma} W^{3}\left\langle\frac{\nabla \gamma}{2 \gamma}, N\right\rangle^{2}=-\left\langle\frac{\nabla \gamma}{2 \gamma}, N\right\rangle^{2} W
$$

and

$$
\begin{aligned}
& -\frac{3}{4} \frac{\left|\nabla^{\Sigma} \gamma\right|^{2}}{4 \gamma^{2}} W-\left\langle\frac{\nabla \gamma}{2 \gamma}, N\right\rangle^{2} W=-\frac{3}{4} \frac{|\nabla \gamma|^{2}}{4 \gamma^{2}} W+\frac{3}{4}\left\langle\frac{\nabla \gamma}{2 \gamma}, N\right\rangle^{2} W-\left\langle\frac{\nabla \gamma}{2 \gamma}, N\right\rangle^{2} W \\
& =-\frac{3}{4} \frac{|\nabla \gamma|^{2}}{4 \gamma^{2}} W-\frac{1}{4}\left\langle\frac{\nabla \gamma}{2 \gamma}, N\right\rangle^{2} W .
\end{aligned}
$$

Hence we obtain

$$
\begin{aligned}
& L W-\frac{2}{W} g^{i j} W_{i} W_{j}=|A|^{2} W+n H W^{3}\left\langle A Y^{T}, Y^{T}\right\rangle-n H W^{3}\left\langle\frac{\nabla \gamma}{2 \gamma^{2}}, N\right\rangle \\
& -3 \gamma\left\langle A Y^{T}, X_{*} \frac{\nabla \gamma}{2 \gamma}\right\rangle+g^{i j} \frac{\gamma_{i ; j}}{2 \gamma} W-\frac{3}{4} \frac{|\nabla \gamma|^{2}}{4 \gamma^{2}} W-\frac{1}{4}\left\langle\frac{\nabla \gamma}{2 \gamma}, N\right\rangle^{2} W \\
& +\gamma W\left\langle\bar{\nabla}_{N} \frac{\bar{\nabla} \gamma}{2 \gamma^{2}}, N\right\rangle-W\left\langle\nabla^{\Sigma} \mathcal{H}, N\right\rangle-\frac{|\nabla \gamma|^{2}}{4 \gamma} \frac{1}{W}-W_{t} .
\end{aligned}
$$

This finishes the proof of the lemma.

\section{REFERENCES}

[1] Maria Calle, Leili Shahriyari, Translating graphs by mean curvature flow in $M^{n} \times \mathbb{R}$. arXiv: 1109.5659v1 [math.DG] (2011)

[2] Bo Guan, Mean curvature motion of nonparametric hypersurfaces with contact angle condition, Elliptic and parabolic methods in geometry (Minneapolis, MN, 1994), A K Peters, Wellesley, MA, 1996, pp. 47-56. MR.1417947(98a:58045)

[3] Gerhard Huisken, Nonparametric mean curvature evolution with boundary conditions, J. Differential Equations 77 (1989), no. 2, 369-378, DOI 10.1016/0022-0396(89)90149-6. MR983300 (90g:35050)

[4] Nicholas J. Korevaar, Maximum principle gradient estimates for the capillary problem, Comm. Partial Differential Equations 13 (1988), no. 1, 1-31, DOI 10.1080/03605308808820536. MR 914812(89d:35061)

[5] Gary M. Lieberman, Second order parabolic differential equations, World Scientific Publishing Co. Inc., River Edge, NJ, 1996. MR.1465184 (98k:35003)

[6] Bo Guan and Joel Spruck, Hypersurfaces of constant mean curvature in hyperbolic space with prescribed asymptotic boundary at infinity, Amer. J. Math. 122 (2000), no. 5, 1039-1060. MR.1781931 (2001j:53069) 
[7] Alexander A. Borisenko and Vicente Miquel, Mean curvature flow of graphs in warped products, Trans. Amer. Math. Soc. 364 (2012), no. 9, 4551-4587, DOI 10.1090/S0002-9947-2012-05425-0. MR2922601

[8] Oliver C. Schnürer and Hartmut R. Schwetlick, Translating solutions for Gauss curvature flows with Neumann boundary conditions, Pacific J. Math. 213 (2004), no. 1, 89-109, DOI 10.2140/pjm.2004.213.89. MR.2040252(2005a:53110)

Departamento de Matemática, Universidade federal do Ceará, Campus do Pici Bloco 914, Fortaleza, Ceará, Brasil 60455-900

E-mail address: jorge.lira@mat.ufc.br

Departamento de Matemática, Universidade Federal da Paraíba, CCEN - Campus i, Jỗo Pessoa, Paraíba, Brasil 58051-900 